\title{
Statistical Mechanics of Financial Markets: \\ Exponential Modifications to Black-Scholes
}

\author{
LESTER INGBER \\ DRW Investments LLC, Chicago Mercantile Exchange Center \\ 30 S Wacker Dr, Ste 1516, Chicago, IL 60606 \\ ingber@ingber.com, ingber@drwtrading.com, ingber@alumni.caltech.edu \\ and \\ JENNIFER K. WILSON \\ DRW Investments LLC, Chicago Mercantile Exchange Center \\ 30 S Wacker Dr, Ste 1516, Chicago, IL 60606 \\ jkw@drwtrading.com
}

\begin{abstract}
The Black-Scholes theory of option pricing has been considered for many years as an important but very approximate zeroth-order description of actual market behavior. We generalize the functional form of the diffusion of these systems and also consider multi-factor models including stochastic volatility. We use a previous development of a statistical mechanics of financial markets to model these issues. Daily Eurodollar futures prices and implied volatilities are fit to determine exponents of functional behavior of diffusions using methods of global optimization, Adaptive Simulated Annealing (ASA), to generate tight fits across moving time windows of Eurodollar contracts. These short-time fitted distributions are then developed into long-time distributions using a robust non-Monte Carlo path-integral algorithm, PATHINT, to generate prices and derivatives commonly used by option traders. The results of our study show that there is only a very small change in at-the money option prices for different probability distributions, both for the one-factor and two-factor models. There still are significant differences in risk parameters, partial derivatives, using more sophisticated models, especially for out-of-the-money options.
\end{abstract}

Keywords: options; eurodollar; volatility; path integral; optimization; statistical mechanics 


\section{INTRODUCTION}

\subsection{Background}

There always is much interest in developing more sophisticated pricing models for financial instruments. In particular, there currently is much interest in improving option pricing models, particularly with respect to stochastic variables [1-4].

The standard Black-Scholes (BS) theory assumes a lognormal distribution of market prices, i.e., a diffusion linearly proportional to the market price. However, many texts include outlines of more general diffusions proportional to an arbitrary power of the market price [5].

The above aspects of stochastic volatility and of more general functional dependencies of diffusions are most often "swept under the rug" of a simple lognormal form. Experienced traders often use their own intuition to put volatility "smiles" into the BS theoretical constant coefficient in the BS lognormal distribution to compensate for these aspects.

It is generally acknowledged that since the market crash of 1987, markets have been increasingly difficult to describe using the BS model, and so better modelling and computational techniques should be used traders [6], although in practice simple BS models are the rule rather than the exception simply because they are easy to use [7]. To a large extent, previous modelling that has included stochastic volatility and multiple factors has been driven more by the desire to either delve into mathematics tangential to these issues, or to deal only with models that can accommodate closed-form algebraic expressions. We do not see much of the philosophy in the literature that has long driven the natural sciences: to respect first raw data, secondly models of raw data, and finally the use of numerical techniques that do not excessively distort models for the sake of ease of analysis and speed of computation. Indeed, very often the reverse set of priorities is seen in mathematical finance.

\subsection{Our Approach}

We address the above issues in detail in this paper within the framework of a previously developed statistical mechanics of financial markets (SMFM) [8-12].

Our approach requires three sensible parts. Part one is the formulation of the model, which to some extent also involves specification of the specific market(s) data to be addressed. Part two is the fitting of 
the model to specific market data. Part three is the use of the resulting model to calculate option prices and their Greeks (partial derivatives of the prices with respect to their independent variables), which are used as risk parameters by traders. Each part requires some specific numerical tuning keeping the real market under consideration. These three parts must be performed in serial. The choice of details for the first part must be made before doing the second part; the choice of details for the second part must be made before doing the third part. Changes in part one require redoing calculations in parts two and three. Changes in part two require redoing calculations in part three.

The first part was to develop the algebraic model to replace/generalize BS, including the possibility of also addressing how to handle data regions not previously observed in trading. This is not absurd; perhaps what is absurd is that current BS models perform integrals that must include a much influence from fat tails that include data regions never seen or likely to be seen in real-world markets. There are some issues as to whether we should take seriously the notion that the market is strongly driven by some element of a "self-fulfilling prophesy" by the BS model [13], but in any case our models have parameters to handle a wide range of possible cases that might arise.

We have developed two parallel tracks starting with part one, a one-factor and a two-factor model. The two-factor model includes stochastic volatility. At first we sensed the need to develop this two-factor model, we now see that this is at the least an important benchmark against which to judge the worth of the one-factor model.

The second part was to fit the actual raw data so we can come up with real distributions. Some tests illustrated that standard quasi-linear fitting routines, even simplex codes, could not get the proper fits, and so we used a more powerful global optimization, Adaptive Simulated Annealing (ASA) [14]. Tuning and selection of the time periods to perform the fits to the data were not trivial aspects of this research. Practical decisions had to be made on the time span of data to be fit and how to aggregate the fits to get sensible "fair values" for reasonable standard deviations of the exponents in the diffusions. Also, recall the serial nature of the parts: As we did fits and learned more about this unchartered area, changes in the models in part one required new fits in part two, etc.

The third part was to develop Greeks and risk parameters from these distributions without making premature approximations just to ease the analysis. Perhaps someday, simple approximations and intuitions similar to what traders now use for BS models will be available for these models, but we do not 
think the best approach is to start out with such approximations until we first see proper calculations, especially in this uncharted territory. When it seemed that Cox-Ross-Rubenstein (CRR) standard tree codes (discretized approximations to partial differential equations) [15] were not stable for general exponents, i.e., for other than the lognormal case, we turned to a PATHINT code developed a decade ago for some hard nonlinear multifactor problems [16], e.g., combat analyses [17], neuroscience [18,19], and potentially chaotic systems [20,21]. In 1990 and 1991 papers on financial applications, it was mentioned how these techniques could be used for stochastic interest rates and bonds [9,10]. The modifications required here for one-factor European and then American cases went surprisingly smoothly; we still had to tune the meshes, etc. The two-factor model presented a technical problem to the algorithm, which we have reasonably handled using a combination of selection of the model in part one (remember the serial nature of the parts ...) and a reasonable approach to developing the meshes. The biggest problem with the two-factor code is that it takes very long to run.

\subsection{Outline of Paper}

Section 1 is this introduction. Section 2 describes the nature of Eurodollar (ED) futures data and the evidence for stochastic volatility. Section 3 outlines the algebra of modelling options, including the standard BS theory and our generalizations. Section 4 outlines the three equivalent mathematical representations used by SMFM; this is required to understand the development of the short-time distribution that defines the cost function we derive for global optimization, as well as the numerical methods we have developed to calculate the long-time evolution of these short-time distributions. Section 5 outlines ASA and explains its use to fit short-time probability distributions defined by our models to the Eurodollar data; we offer the fitted exponent in the diffusion as a new important technical indicator of market behavior. Section 6 outlines PATHINT and explains its use to develop long-time probability distributions from the fitted short-time probability distributions, for both the one-factor and two-factor tracks. Section 7 describes how we use these long-time probability distributions to calculate European and American option prices and Greeks; here we give numerical tests of our approach to BS CRR algorithms. Section 8 is our conclusion. 


\section{DATA}

\subsection{Eurodollars}

Eurodollars are fixed-rate time deposits held primarily by overseas banks, but denominated in US dollars. They are not subject to US banking regulations and therefore tend to have a tighter bid-ask spread than deposits held in the United States [22].

\subsection{Futures}

The three-month Eurodollar futures contract is one of the most actively traded futures markets in the world. The contract is quoted as an index where the yield is equal to the Eurodollar price subtracted

from 100. This yield is equal to the fixed rate of interest paid by Eurodollar time deposits upon maturity and is expressed as an annualized interest rate based on a 360-day year. The Eurodollar futures are cash settled based on the 90-day London Interbank Offer Rate (LIBOR). A “notional” principal amount of \$1 million, is used to determine the change in the total interest payable on a hypothetical underlying time deposit, but is never actually paid or received [22].

Currently a total of 40 quarterly Eurodollar futures contracts (or ten years worth) are listed, with expirations annually in March, June, September and December.

\subsection{Options on Futures}

The options traded on the Eurodollar futures include not only 18 months of options expiring at the same time as the underlying future, but also various short dated options which themselves expire up to one year prior to the expiration of the underlying futures contract.

\subsection{Front/Back Month Contracts}

For purposes of risk minimization, as discussed in a previous paper [4], traders put on spreads across a variety of option contracts. One common example is to trade the spread on contracts expiring one year apart, where the future closer to expiration is referred to as the front month contract, and the future expiring one year later is called the back month. The availability of short dated or "mid-curve" options which are based on an underlying back month futures contract, but expire at the same time as the 
front month, allow one to trade the volatility ratios of the front and back month futures contracts without having to take the time differences in option expirations into consideration. We studied the volatilities of these types of front and back month contracts. Here, we give analyses with respect only to quarterly data longer than six months from expiration.

\subsection{Stochastic Volatility}

Below we develop two-factor models to address stochastic volatility. In a previous paper, we have performed empirical studies of Eurodollar futures to support the necessity of dealing with these issues [4].

\subsubsection{Eurodollar Volatility of Historical Volatility}

Fig. 1(a) gives a comparison of Basis-Point Volatility (BPV), standard deviation of BPV (SDBPV), and standard deviation of differenced BPV (SDDBPV), for Front and Back contracts, marked to the number of days to expiration of the Front contract, for years 1995, 1996, and 1997, respectively. The SDBPV illustrate that there exists a distribution of volatilities about the mean volatility. The SDDBPV illustrate that this distribution likely is a stochastic process with a constant diffusion.

Fig. 1(b) gives a comparison of standard deviation of differenced Basis-Point Volatility (SDDBPV) with and standard deviation of differenced Black-Scholes Volatility (SDDBSV), for Front and Back contracts. The SDDBSV have been scaled to the SDDBPV by multiplying them by the rounded average of the yields, i.e., 6.0. Note that after scaling, they consistently lie close to each other. Thus, both the BPV and Black-Scholes Volatility (BSV) have volatilities that can be considered to be stochastic processes with constant diffusion.

Fig. 1.

\subsubsection{Eurodollar Volatility of Implied Volatility}

Fig. 1(c) shows the Basis-Point Implied Volatility (BPIV) of Front and Back contracts.

Fig. 1(d) shows the standard deviation of differenced Basis-Point Implied Volatility (SDDBPIV) of Front and Back contracts. Note that, similar to the results with historical volatilities, this illustrates that 
the volatility of the implied volatilities appears to be a stochastic process with constant diffusion for times less than 30 days before expiration; afterwards, the values still are within the same scale.

\section{MODELS}

\subsection{Random walk model}

The use of Brownian motion as a model for financial systems is generally attributed to Bachelier [23], though he incorrectly intuited that the noise scaled linearly instead of as the square root relative to the random log-price variable. Einstein is generally credited with using the correct mathematical description in a larger physical context of statistical systems. However, several studies imply that changing prices of many markets do not follow a random walk, that they may have long-term dependences in price correlations, and that they may not be efficient in quickly arbitraging new

information [24-26]. A random walk for returns, rate of change of prices over prices, is described by a Langevin equation with simple additive noise $\eta$, typically representing the continual random influx of information into the market.

$$
\begin{aligned}
& \dot{\Gamma}=-\gamma_{1}+\gamma_{2} \eta, \\
& \dot{\Gamma}=d \Gamma / d t, \\
& <\eta(t)>_{\eta}=0,\left\langle\eta(t), \eta\left(t^{\prime}\right)>_{\eta}=\delta\left(t-t^{\prime}\right),\right.
\end{aligned}
$$

where $\gamma_{1}$ and $\gamma_{2}$ are constants, and $\Gamma$ is the logarithm of (scaled) price. Price, although the most dramatic observable, may not be the only appropriate dependent variable or order parameter for the system of markets [27]. This possibility has also been called the "semistrong form of the efficient market hypothesis" [24].

The generalization of this approach to include multivariate nonlinear nonequilibrium markets led to a model of statistical mechanics of financial markets (SMFM) [8].

\subsection{Black-Scholes (BS) Theory}

The standard partial-differential equation used to formulate most variants of Black-Scholes (BS) models describing the market value of an option, $V$, is 


$$
\frac{\partial V}{\partial t}+\frac{1}{2} \sigma^{2} S^{2} \frac{\partial^{2} V}{\partial S^{2}}+r S \frac{\partial V}{\partial S}-r V=0
$$

where $S$ is the asset price, and $\sigma$ is the standard deviation, or volatility of $S$, and $r$ is the short-term interest rate. The solution depends on boundary conditions, subject to a number of interpretations, some requiring minor transformations of the basic BS equation or its solution. For example, the basic equation can apply to a number of one-dimensional models of interpretations of prices given to $V$, e.g., puts or calls, and to $S$, e.g., stocks or futures, dividends, etc.

For instance, if $V$ is set to $C$, a call on an European option with exercise price $E$ with maturity at $T$, the solution is

$$
\begin{aligned}
& C(S, t)=S N\left(d_{1}\right)-E e^{-r(T-t)} N\left(d_{2}\right), \\
& d_{1}=\frac{\ln (S / E)+\left(r+\frac{1}{2} \sigma^{2}\right)(T-t)}{\sigma(T-t)^{1 / 2}}, \\
& d_{2}=\frac{\ln (S / E)+\left(r-\frac{1}{2} \sigma^{2}\right)(T-t)}{\sigma(T-t)^{1 / 2}} .
\end{aligned}
$$

In practice, the volatility $\sigma$ is the least known parameter in this equation, and its estimation is generally the most important part of pricing options. Usually the volatility is given in a yearly basis, baselined to some standard, e.g., 252 trading days per year, or 360 or 365 calendar days. Therefore, all values of volatility given in the graphs in this paper, based on daily data, would be annualized by multiplying the standard deviations of the yields by $\sqrt{252}=15.87$. We have used this factor to present our implied volatilities as daily movements.

\subsection{Some Key Issues in Derivation of BS}

The basic BS model considers a portfolio in terms of delta $(\Delta)$,

$$
\Pi=V-\Delta S
$$

in a market with Gaussian-Markovian ("white") noise $X$ and drift $\mu$,

$$
\frac{d S}{S}=\sigma d X+\mu d t
$$


where $V(S, t)$ inherits a random process from $S$,

$$
d V=\sigma S \frac{\partial V}{\partial S} d X+\left(\mu S \frac{\partial V}{\partial S}+\frac{1}{2} \sigma^{2} S^{2} \frac{\partial^{2} V}{\partial S^{2}}+\frac{\partial V}{\partial t}\right) d t
$$

This yields

$$
d \Pi=\sigma\left(\frac{\partial V}{\partial S}-\Delta\right) d X+\left(\mu S \frac{\partial V}{\partial S}+\frac{1}{2} \sigma^{2} S^{2} \frac{\partial^{2} V}{\partial S^{2}}+\frac{\partial V}{\partial t}-\mu \Delta S\right) d t
$$

The expected risk-neutral return of $\Pi$ is

$$
d \Pi=r \Pi d t=r(V-\Delta S) d t .
$$

Options $V$ on futures $F$ can be derived, e.g., using simple transformations to take cost of carry into consideration, such as

$$
F=S e^{r(T-t)},
$$

and setting

$$
d \Pi=r V d t .
$$

The corresponding BS equation for futures $F$ is

$$
\frac{\partial V}{\partial t}+\frac{1}{2} \sigma^{2} F^{2} \frac{\partial^{2} V}{\partial S^{2}}-r V=0
$$

At least two advantages are present if $\Delta$ is chosen such that

$$
\Delta=\frac{\partial V}{\partial S} .
$$

Then, the portfolio can be instantaneously "risk-neutral," in terms of zeroing the coefficient of $X$, as well as independent of the direction of market, in terms of zeroing the coefficient of $\mu$. For the above example of $V=C$,

$$
\Delta=N\left(d_{1}\right)
$$

Other trading strategies based on this simple model use similar constructs as risk parameters, e.g., gamma $(\Gamma)$, theta $(\Theta)$, vega, rho $(\rho)[5]$, 


$$
\begin{gathered}
\Gamma=\frac{\partial^{2} \Pi}{\partial S^{2}}, \\
\Theta=\frac{\partial \Pi}{\partial t}, \\
\operatorname{vega}=\frac{\partial \Pi}{\partial \sigma}, \\
\rho=\frac{\partial \Pi}{\partial r} .
\end{gathered}
$$

The BS equation, Eq. (2), may be written as

$$
\Theta+r S \Delta+\frac{1}{2}(\sigma S)^{2} \Gamma=r f
$$

\subsection{Some Generalizations of BS}

The volatility $\sigma$ may depend on other variables, and the BS model may be generalized to multivariable models. However, within the framework of the basic BS model, if $\sigma$ and $r$ are timedependent, then it turns out that the above solutions of the basic BS, and the use of the above set of $\{\Delta, \Gamma, \Theta$, vega, $\rho\}$, etc., can be used without change, provided an "effective" volatility, $\hat{\sigma}$, is defined in terms of $\sigma(t)$, and an "effective" interest-rate, $\hat{r}$, is defined in terms of $r(t)$, are defined.

This can be developed by considering a slight generalization of the above BS equation for the variable $\hat{V}$, using methods given in a standard text [28]. A transformation of variables is used to transform away any time-dependent coefficients,

$$
\begin{aligned}
& \hat{S}=S e^{\alpha(t)}, \\
& \hat{V}=V e^{\beta(t)}, \\
& \hat{t}=\gamma(t) .
\end{aligned}
$$

This leads to

$$
\frac{d \gamma(t)}{d t} \frac{\partial \hat{V}}{\partial \hat{t}}+\frac{1}{2} \sigma(t)^{2} \hat{S}^{2} \frac{\partial^{2} \hat{V}}{\partial \hat{S}^{2}}+\left(r(t)+\frac{d \alpha(t)}{d t}\right) \hat{S} \frac{\partial \hat{V}}{\partial \hat{S}}-\left(r(t)+\frac{d \beta(t)}{d t}\right) \hat{V}=0
$$




$$
\begin{aligned}
& \alpha(t)=\int_{t}^{T} r(\tau) d \tau, \\
& \beta(t)=\int_{t}^{T} r(\tau) d \tau, \\
& \gamma(t)=\int_{t}^{T} \sigma^{2}(\tau) d \tau,
\end{aligned}
$$

leads to

$$
\frac{\partial \hat{V}}{\partial \hat{t}}=\frac{1}{2} \hat{S}^{2} \frac{\partial^{2} \hat{V}}{\partial \hat{S}^{2}},
$$

with coefficients independent of time $t$ (the motivation for this transformation). In terms of $V$,

$$
V(S, t)=e^{-\beta(t)} \hat{V}\left(S e^{\alpha(t)}, \gamma(t)\right)
$$

To get the explicit form of the solution, consider the original BS model with constant coefficients and solution $V_{B S}$ :

$$
\left.V_{B S}=e^{-(T-t) r} \hat{V}_{B S}\left(S e^{-(T-t) r},(T-t) \sigma^{2}\right)\right)
$$

for some solution $\hat{V}_{B S}$. Therefore, the standard BS solutions for various products can be used if $\sigma$ and/or $r$ are time-dependent, by replacing $\sigma$ in the original equation by $\hat{\sigma}$, and by replacing $r$ in the original equation by $\hat{r}$,

$$
\begin{gathered}
\sigma^{2} \rightarrow \hat{\sigma}^{2}=\frac{1}{T-t} \int_{t}^{T} \sigma^{2}(\tau) d \tau, \\
r \rightarrow \hat{r}=\frac{1}{T-t} \int_{t}^{T} r(\tau) d \tau .
\end{gathered}
$$




\section{5. $S^{x}$ Models}

Our two-factor model includes stochastic volatility $\sigma$ of the underlying $S$,

$$
\begin{aligned}
& d S=\mu d t+\sigma F\left(S, S_{0}, S_{\infty}, x, y\right) d z_{S} \\
& d \sigma=v d t+\varepsilon d z_{\sigma} \\
& <d z_{i}>=0, i=\{S, \sigma\} \\
& <d z_{i}(t) d z_{j}\left(t^{\prime}\right)>=d t \delta\left(t-t^{\prime}\right), i=j \\
& <d z_{i}(t) d z_{j}\left(t^{\prime}\right)>=\rho d t \delta\left(t-t^{\prime}\right), i \neq j \\
& F\left(S, S_{0}, S_{\infty}, x, y\right)= \begin{cases}S, & S<S_{0} \\
S^{x} S_{0}^{1-x}, & S_{0} \leq S \leq S_{\infty} \\
S^{y} S_{0}^{1-x} S_{\infty}^{x-y}, & S>S_{\infty}\end{cases}
\end{aligned}
$$

where $S_{0}$ and $S_{\infty}$ are selected to lie outside the data region used to fit the other parameters, e.g., $S_{0}=1 / 2$ and $S_{\infty}=20$ for fits to Eurodollar futures which historically have a very tight range relative to other markets. We have used the Black-Scholes form $F=S$ inside $S<S_{0}$ to obtain the usual benefits, e.g., no negative prices as the distribution is naturally excluded from $S<0$ and preservation of put-call parity. Put-call parity for European options is derived quite independent of any mathematical model of options [5]. In its simplest form, it is given by

$$
c+X e^{-r(T-t)}=p+S
$$

where $c(p)$ is the fair price of a call (put), $X$ is the strike price, $r$ is the risk-free interest rate, $t$ is the present time, $T$ is the time of expiration, and $S$ is the underlying market. We have taken $y=0$, a normal distribution, to reflect total ignorance of markets outside the range of $S>S_{\infty}$. The one-factor model just assumes a constant $\sigma$. It is often noted that BS models incorrectly include untenable contributions from large $S$ regions because of their fat tails [29]. (If we wished to handle negative interest rates, ED prices > 100 , we would move shift the $S=0$ axis to some $S<0$ value.)

We found that the abrupt, albeit continuous, changes across $S_{0}$ especially for $x \leq 0$ did not cause any similar effects in the distributions evolved using these diffusions, as reported below. 
The formula for pricing an option $P$, derived in a Black-Scholes generalized framework after factoring out interest-rate discounting, is equivalent to using the form

$$
\begin{aligned}
& d S=\mu S d t+\sigma F\left(S, S_{0}, S_{\infty}, x, y\right) d z_{S} \\
& d \sigma=v d t+\varepsilon d z_{\sigma}
\end{aligned}
$$

We experimented with some alternative functional forms, primarily to apply some smooth cutoffs across the above three regions of $S$. For example, we used $F^{\prime}$, a function $F$ designed to revert to the lognormal Black-Scholes model in several limits,

$$
\begin{aligned}
& F^{\prime}\left(S, S_{0}, S_{\infty}, x\right)=S C_{0}+\left(1-C_{0}\right)\left(S^{x} S_{0}^{1-x} C_{\infty}+S_{0}\left(1-C_{\infty}\right)\right) \\
& C_{0}=\exp \left[-\left(\frac{S}{S_{0}} \frac{|1-x|}{1+|1-x|}\right)^{|2-x|+1}\right] \\
& C_{\infty}=\exp \left[-\left(\frac{S}{S_{\infty}}\right)^{2}\right] \\
& \lim _{S \rightarrow \infty, x \neq 1} F^{\prime}\left(S, S_{0}, S_{\infty}, x\right)=S_{0}=\text { constant } \\
& \lim _{S \rightarrow 0^{+}} F^{\prime}\left(S, S_{0}, S_{\infty}, x\right)=\lim _{x \rightarrow 1} F^{\prime}\left(S, S_{0}, S_{\infty}, x\right)=S
\end{aligned}
$$

However, our fits were most sensitive to the data when we permitted the central region to be pure $S^{x}$ using $F$ above.

\subsubsection{Various $F(S, x)$ Diffusions}

Fig. 2 gives examples of $F\left(S, S_{0}, S_{\infty}, x, y\right) d z_{S}$ for $x$ in $\{-1,0,1,2\}$. The other parameters are $S=5, S_{0}=0.5, S_{\infty}=20, y=0$.

Fig. 2. 


\section{STATISTICAL MECHANICS OF FINANCIAL MARKETS (SMFM)}

\subsection{Statistical Mechanics of Large Systems}

Aggregation problems in nonlinear nonequilibrium systems typically are "solved" (accommodated) by having new entities/languages developed at these disparate scales in order to efficiently pass information back and forth. This is quite different from the nature of quasi-equilibrium quasi-linear systems, where thermodynamic or cybernetic approaches are possible. These approaches typically fail for nonequilibrium nonlinear systems.

Many systems are aptly modeled in terms of multivariate differential rate-equations, known as Langevin equations,

$$
\begin{aligned}
& \dot{M}^{G}=f^{G}+\hat{g}_{j}^{G} \eta^{j},(G=1, \cdots, \Lambda),(j=1, \cdots, N), \\
& \dot{M}^{G}=d M^{G} / d t, \\
& <\eta^{j}(t)>_{\eta}=0,<\eta^{j}(t), \eta^{j^{\prime}}\left(t^{\prime}\right)>_{\eta}=\delta^{j j^{\prime}} \delta\left(t-t^{\prime}\right),
\end{aligned}
$$

where $f^{G}$ and $\hat{g}_{j}^{G}$ are generally nonlinear functions of mesoscopic order parameters $M^{G}, j$ is a microscopic index indicating the source of fluctuations, and $N \geq \Lambda$. The Einstein convention of summing over repeated indices is used. Vertical bars on an index, e.g., |j|, imply no sum is to be taken on repeated indices.

Via a somewhat lengthy, albeit instructive calculation, outlined in several other papers $[8,10,30]$, involving an intermediate derivation of a corresponding Fokker-Planck or Schrodinger-type equation for the conditional probability distribution $P\left[M(t) \mid M\left(t_{0}\right)\right]$, the Langevin rate Eq. (27) is developed into the more useful probability distribution for $M^{G}$ at long-time macroscopic time event $t=(u+1) \theta+t_{0}$, in terms of a Stratonovich path-integral over mesoscopic Gaussian conditional probabilities [31-35]. Here, macroscopic variables are defined as the long-time limit of the evolving mesoscopic system.

The corresponding Schrodinger-type equation is [33,34]

$$
\begin{aligned}
& \partial P / \partial t=\frac{1}{2}\left(g^{G G^{\prime}} P\right)_{, G G^{\prime}}-\left(g^{G} P\right)_{, G}+V, \\
& g^{G G^{\prime}}=k_{T} \delta^{j k} \hat{g}_{j}^{G} \hat{g}_{k}^{G^{\prime}},
\end{aligned}
$$




$$
\begin{aligned}
& g^{G}=f^{G}+\frac{1}{2} \delta^{j k} \hat{g}_{j}^{G^{\prime}} \hat{g}_{k, G^{\prime}}^{G}, \\
& {[\cdots]_{, G}=\partial[\cdots] / \partial M^{G} .}
\end{aligned}
$$

This is properly referred to as a Fokker-Planck equation when $V \equiv 0$. Note that although the partial differential Eq. (28) contains equivalent information regarding $M^{G}$ as in the stochastic differential Eq. (27), all references to $j$ have been properly averaged over. I.e., $\hat{g}_{j}^{G}$ in Eq. (27) is an entity with parameters in both microscopic and mesoscopic spaces, but $M$ is a purely mesoscopic variable, and this is more clearly reflected in Eq. (28).

The path integral representation is given in terms of the Lagrangian $L$.

$$
\begin{aligned}
& P\left[M_{t} \mid M_{t_{0}}\right] d M(t)=\int \cdots \int \underline{D} M \exp (-S) \delta\left[M\left(t_{0}\right)=M_{0}\right] \delta\left[M(t)=M_{t}\right], \\
& S=k_{T}^{-1} \min \int_{t_{0}}^{t} d t^{\prime} L \\
& \underline{D} M=\lim _{u \rightarrow \infty} \prod_{\rho=1}^{u+1} g^{1 / 2} \prod_{G}(2 \pi \theta)^{-1 / 2} d M_{\rho}^{G} \\
& L\left(\dot{M}^{G}, M^{G}, t\right)=\frac{1}{2}\left(\dot{M}^{G}-h^{G}\right) g_{G G^{\prime}}\left(\dot{M}^{G^{\prime}}-h^{G^{\prime}}\right)+\frac{1}{2} h_{; G}^{G}+R / 6-V, \\
& h^{G}=g^{G}-\frac{1}{2} g^{-1 / 2}\left(g^{1 / 2} g^{G G^{\prime}}\right)_{G^{\prime}}, \\
& g_{G G^{\prime}}=\left(g^{G G^{\prime}}\right)^{-1}, \\
& g=\operatorname{det}\left(g_{G G^{\prime}}\right) \\
& h_{; G}^{G}=h_{, G}^{G}+\Gamma_{G F}^{F} h^{G}=g^{-1 / 2}\left(g^{1 / 2} h^{G}\right)_{, G}, \\
& \Gamma_{J K}^{F} \equiv g^{L F}[J K, L]=g^{L F}\left(g_{J L, K}+g_{K L, J}-g_{J K, L}\right), \\
& R=g^{J L} R_{J L}=g^{J L} g^{J K} R_{F J K L}, \\
& R_{F J K L}=\frac{1}{2}\left(g_{F K, J L}-g_{J K, F L}-g_{F L, J K}+g_{J L, F K}\right)+g_{M N}\left(\Gamma_{F K}^{M} \Gamma_{J L}^{N}-\Gamma_{F L}^{M} \Gamma_{J K}^{N}\right) .
\end{aligned}
$$


Mesoscopic variables have been defined as $M^{G}$ in the Langevin and Fokker-Planck representations, in terms of their development from the microscopic system labeled by $j$. The Riemannian curvature term $R$ arises from nonlinear $g_{G G^{\prime}}$, which is a bona fide metric of this space [33]. Even if a stationary solution, i.e., $\dot{M}^{G}=0$, is ultimately sought, a necessarily prior stochastic treatment of $\dot{M}^{G}$ terms gives rise to these Riemannian "corrections." Even for a constant metric, the term $h_{; G}^{G}$ contributes to $L$ for a nonlinear mean $h^{G}$. $V$ may include terms such as $\sum_{T^{\prime}} J_{T^{\prime} G} M^{G}$, where the Lagrange multipliers $J_{T^{\prime} G}$ are constraints on $M^{G}$, which are advantageously modeled as extrinsic sources in this representation; they too may be time-dependent. Using the variational principle, $J_{T G}$ may also be used to constrain $M^{G}$ to regions where they are empirically bound. More complicated constraints may be affixed to $L$ using methods of optimal control theory [36]. With respect to a steady state $\bar{P}$, when it exists, the information gain in state $P$ is defined by

$$
\begin{aligned}
& \Upsilon[P]=\int \cdots \int \underline{D} M^{\prime} P \ln (P / \bar{P}), \\
& \underline{D} M^{\prime}=\underline{D} M / d M_{u+1} .
\end{aligned}
$$

In the economics literature, there appears to be sentiment to define Eq. (27) by the Ito, rather than the Stratonovich prescription. It is true that Ito integrals have Martingale properties not possessed by Stratonovich integrals [37] which leads to risk-neural theorems for markets [38,39], but the nature of the proper mathematics should eventually be determined by proper aggregation of relatively microscopic models of markets. It should be noted that virtually all investigations of other physical systems, which are also continuous time models of discrete processes, conclude that the Stratonovich interpretation coincides with reality, when multiplicative noise with zero correlation time, modeled in terms of white noise $\eta^{j}$, is properly considered as the limit of real noise with finite correlation time [40]. The path integral succinctly demonstrates the difference between the two: The Ito prescription corresponds to the prepoint discretization of $L$, wherein $\theta \dot{M}(t) \rightarrow M_{\rho+1}-M_{\rho}$ and $M(t) \rightarrow M_{\rho}$. The Stratonovich prescription corresponds to the midpoint discretization of $L$, wherein $\theta \dot{M}(t) \rightarrow M_{\rho+1}-M_{\rho}$ and $M(t) \rightarrow \frac{1}{2}\left(M_{\rho+1}+M_{\rho}\right)$. In terms of the functions appearing in the Fokker-Planck Eq. (28), the Ito prescription of the prepoint discretized Lagrangian, $L_{I}$, is relatively simple, albeit deceptively so because of its nonstandard calculus. 


$$
L_{I}\left(\dot{M}^{G}, M^{G}, t\right)=\frac{1}{2}\left(\dot{M}^{G}-g^{G}\right) g_{G G^{\prime}}\left(\dot{M}^{G^{\prime}}-g^{G^{\prime}}\right)-V
$$

In the absence of a nonphenomenological microscopic theory, the difference between a Ito prescription and a Stratonovich prescription is simply a transformed drift [41].

There are several other advantages to Eq. (29) over Eq. (27). Extrema and most probable states of $M^{G}, \ll M^{G} \gg$, are simply derived by a variational principle, similar to conditions sought in previous studies [42]. In the Stratonovich prescription, necessary, albeit not sufficient, conditions are given by

$$
\begin{aligned}
& \delta_{G} L=L_{, G}-L_{, \dot{G}: t}=0, \\
& L_{, \dot{G}: t}=L_{, \dot{G} G^{\prime}} \dot{M}^{G^{\prime}}+L_{, \dot{G} \dot{G}^{\prime}} \ddot{M}^{G^{\prime}} .
\end{aligned}
$$

For stationary states, $\dot{M}^{G}=0$, and $\partial \bar{L} / \partial \bar{M}^{G}=0$ defines $\ll \bar{M}^{G} \gg$, where the bars identify stationary variables; in this case, the macroscopic variables are equal to their mesoscopic counterparts. [Note that $\bar{L}$ is not the stationary solution of the system, e.g., to Eq. (28) with $\partial P / \partial t=0$. However, in some cases [43], $\bar{L}$ is a definite aid to finding such stationary states.] Many times only properties of stationary states are examined, but here a temporal dependence is included. E.g., the $\dot{M}^{G}$ terms in $L$ permit steady states and their fluctuations to be investigated in a nonequilibrium context. Note that Eq. (32) must be derived from the path integral, Eq. (29), which is at least one reason to justify its development.

\subsection{Algebraic Complexity Yields Simple Intuitive Results}

It must be emphasized that the output need not be confined to complex algebraic forms or tables of numbers. Because $L$ possesses a variational principle, sets of contour graphs, at different long-time epochs of the path-integral of $P$ over its variables at all intermediate times, give a visually intuitive and accurate decision-aid to view the dynamic evolution of the scenario. For example, this Lagrangian approach permits a quantitative assessment of concepts usually only loosely defined.

$$
\begin{aligned}
& \text { "Momentum" }=\Pi^{G}=\frac{\partial L}{\partial\left(\partial M^{G} / \partial t\right)}, \\
& \text { "Mass" } g_{G G^{\prime}}=\frac{\partial^{2} L}{\partial\left(\partial M^{G} / \partial t\right) \partial\left(\partial M^{G^{\prime}} / \partial t\right)}, \\
& \text { "Force" }=\frac{\partial L}{\partial M^{G}},
\end{aligned}
$$




$$
\text { “F }=m a ": \delta L=0=\frac{\partial L}{\partial M^{G}}-\frac{\partial}{\partial t} \frac{\partial L}{\partial\left(\partial M^{G} / \partial t\right)},
$$

where $M^{G}$ are the variables and $L$ is the Lagrangian. These physical entities provide another form of intuitive, but quantitatively precise, presentation of these analyses. For example, daily newspapers use some of this terminology to discuss the movement of security prices. In this paper, the $\Pi^{G}$ serve as canonical momenta indicators (CMI) for these systems.

\subsubsection{Derived Canonical Momenta Indicators (CMI)}

Because $L$ possesses a variational principle, sets of contour graphs, at different long-time epochs of the path-integral of $P$ over its variables at all intermediate times, give a visually intuitive and accurate decision-aid to view the dynamic evolution of the scenario.

The extreme sensitivity of the CMI gives rapid feedback on changes in trends as well as the volatility of markets, and therefore are good indicators to use for trading rules [12]. A time-locked moving average provides manageable indicators for trading signals. This is a current project using such CMI developed as a byproduct of the ASA fits described below.

\section{ADAPTIVE SIMULATED ANNEALING (ASA) FITS}

\subsection{ASA Outline}

The algorithm developed which is now called Adaptive Simulated Annealing (ASA) [44] fits shorttime probability distributions to observed data, using a maximum likelihood technique on the Lagrangian. This algorithm has been developed to fit observed data to a theoretical cost function over a $D$-dimensional parameter space [44], adapting for varying sensitivities of parameters during the fit. The ASA code can be obtained at no charge, via WWW from http://www.ingber.com/ or via FTP from ftp.ingber.com.

\subsubsection{General description}

Simulated annealing (SA) was developed in 1983 to deal with highly nonlinear problems [45], as an extension of a Monte-Carlo importance-sampling technique developed in 1953 for chemical physics problems. It helps to visualize the problems presented by such complex systems as a geographical terrain. 
For example, consider a mountain range, with two "parameters," e.g., along the North-South and East-West directions. We wish to find the lowest valley in this terrain. SA approaches this problem similar to using a bouncing ball that can bounce over mountains from valley to valley. We start at a high "temperature," where the temperature is an SA parameter that mimics the effect of a fast moving particle in a hot object like a hot molten metal, thereby permitting the ball to make very high bounces and being able to bounce over any mountain to access any valley, given enough bounces. As the temperature is made relatively colder, the ball cannot bounce so high, and it also can settle to become trapped in relatively smaller ranges of valleys.

We imagine that our mountain range is aptly described by a "cost function." We define probability distributions of the two directional parameters, called generating distributions since they generate possible valleys or states we are to explore. We define another distribution, called the acceptance distribution, which depends on the difference of cost functions of the present generated valley we are to explore and the last saved lowest valley. The acceptance distribution decides probabilistically whether to stay in a new lower valley or to bounce out of it. All the generating and acceptance distributions depend on temperatures.

In 1984 [46], it was established that SA possessed a proof that, by carefully controlling the rates of cooling of temperatures, it could statistically find the best minimum, e.g., the lowest valley of our example above. This was good news for people trying to solve hard problems which could not be solved by other algorithms. The bad news was that the guarantee was only good if they were willing to run SA forever. In 1987, a method of fast annealing (FA) was developed [47], which permitted lowering the temperature exponentially faster, thereby statistically guaranteeing that the minimum could be found in some finite time. However, that time still could be quite long. Shortly thereafter, Very Fast Simulated Reannealing (VFSR) was developed in 1987 [44], now called Adaptive Simulated Annealing (ASA), which is exponentially faster than FA.

ASA has been applied to many problems by many people in many disciplines [48-50]. The feedback of many users regularly scrutinizing the source code ensures its soundness as it becomes more flexible and powerful. 


\subsubsection{Mathematical outline}

ASA considers a parameter $\alpha_{k}^{i}$ in dimension $i$ generated at annealing-time $k$ with the range

$$
\alpha_{k}^{i} \in\left[A_{i}, B_{i}\right]
$$

calculated with the random variable $y^{i}$,

$$
\begin{aligned}
& \alpha_{k+1}^{i}=\alpha_{k}^{i}+y^{i}\left(B_{i}-A_{i}\right), \\
& y^{i} \in[-1,1] .
\end{aligned}
$$

The generating function $g_{T}(y)$ is defined,

$$
g_{T}(y)=\prod_{i=1}^{D} \frac{1}{2\left(\left|y^{i}\right|+T_{i}\right) \ln \left(1+1 / T_{i}\right)} \equiv \prod_{i=1}^{D} g_{T}^{i}\left(y^{i}\right)
$$

where the subscript $i$ on $T_{i}$ specifies the parameter index, and the $k$-dependence in $T_{i}(k)$ for the annealing schedule has been dropped for brevity. Its cumulative probability distribution is

$$
\begin{aligned}
& G_{T}(y)=\int_{-1}^{y^{1}} \cdots \int_{-1}^{y^{D}} d y^{\prime 1} \cdots d y^{D} g_{T}\left(y^{\prime}\right) \equiv \prod_{i=1}^{D} G_{T}^{i}\left(y^{i}\right), \\
& G_{T}^{i}\left(y^{i}\right)=\frac{1}{2}+\frac{\operatorname{sgn}\left(y^{i}\right)}{2} \frac{\ln \left(1+\left|y^{i}\right| / T_{i}\right)}{\ln \left(1+1 / T_{i}\right)} .
\end{aligned}
$$

$y^{i}$ is generated from a $u^{i}$ from the uniform distribution

$$
\begin{aligned}
& u^{i} \in U[0,1], \\
& y^{i}=\operatorname{sgn}\left(u^{i}-\frac{1}{2}\right) T_{i}\left[\left(1+1 / T_{i}\right)^{\left|2 u^{i}-1\right|}-1\right] .
\end{aligned}
$$

It is straightforward to calculate that for an annealing schedule for $T_{i}$

$$
T_{i}(k)=T_{0 i} \exp \left(-c_{i} k^{1 / D}\right)
$$

a global minima statistically can be obtained. I.e.,

$$
\sum_{k_{0}}^{\infty} g_{k} \approx \sum_{k_{0}}^{\infty}\left[\prod_{i=1}^{D} \frac{1}{2\left|y^{i}\right| c_{i}}\right] \frac{1}{k}=\infty
$$

Control can be taken over $c_{i}$, such that 


$$
\begin{aligned}
& T_{f i}=T_{0 i} \exp \left(-m_{i}\right) \text { when } k_{f}=\exp n_{i}, \\
& c_{i}=m_{i} \exp \left(-n_{i} / D\right),
\end{aligned}
$$

where $m_{i}$ and $n_{i}$ can be considered "free" parameters to help tune ASA for specific problems.

\subsubsection{Reannealing}

ASA has over 100 OPTIONS available for tuning. A few important ones are described here.

Whenever doing a multi-dimensional search in the course of a complex nonlinear physical problem, inevitably one must deal with different changing sensitivities of the $\alpha^{i}$ in the search. At any given annealing-time, the range over which the relatively insensitive parameters are being searched can be "stretched out" relative to the ranges of the more sensitive parameters. This can be accomplished by periodically rescaling the annealing-time $k$, essentially reannealing, every hundred or so acceptanceevents (or at some user-defined modulus of the number of accepted or generated states), in terms of the sensitivities $s_{i}$ calculated at the most current minimum value of the cost function, $C$,

$$
s_{i}=\partial C / \partial \alpha^{i}
$$

In terms of the largest $s_{i}=s_{\max }$, a default rescaling is performed for each $k_{i}$ of each parameter dimension, whereby a new index $k_{i}^{\prime}$ is calculated from each $k_{i}$,

$$
\begin{aligned}
& k_{i} \rightarrow k_{i}^{\prime}, \\
& {T^{\prime}}_{i k^{\prime}}=T_{i k}\left(s_{\max } / s_{i}\right), \\
& {k^{\prime}}_{i}=\left(\ln \left(T_{i 0} / T_{i k^{\prime}}\right) / c_{i}\right)^{D} .
\end{aligned}
$$

$T_{i 0}$ is set to unity to begin the search, which is ample to span each parameter dimension.

\subsubsection{Quenching}

Another adaptive feature of ASA is its ability to perform quenching in a methodical fashion. This is applied by noting that the temperature schedule above can be redefined as

$$
\begin{aligned}
& T_{i}\left(k_{i}\right)=T_{0 i} \exp \left(-c_{i} k_{i}^{Q_{i} / D}\right), \\
& c_{i}=m_{i} \exp \left(-n_{i} Q_{i} / D\right),
\end{aligned}
$$


in terms of the "quenching factor" $Q_{i}$. The sampling proof fails if $Q_{i}>1$ as

$$
\sum_{k} \prod^{D} 1 / k^{Q_{i} / D}=\sum_{k} 1 / k^{Q_{i}}<\infty
$$

This simple calculation shows how the "curse of dimensionality" arises, and also gives a possible way of living with this disease. In ASA, the influence of large dimensions becomes clearly focussed on the exponential of the power of $k$ being $1 / D$, as the annealing required to properly sample the space becomes prohibitively slow. So, if resources cannot be committed to properly sample the space, then for some systems perhaps the next best procedure may be to turn on quenching, whereby $Q_{i}$ can become on the order of the size of number of dimensions.

The scale of the power of $1 / D$ temperature schedule used for the acceptance function can be altered in a similar fashion. However, this does not affect the annealing proof of ASA, and so this may used without damaging the sampling property.

\subsubsection{Widespread use and comparisons}

The file http://www.ingber.com/MISC.DIR/asa_examples has several templates of "toy" test problems, especially illustrating how tuning can increase the efficiency of ASA by orders of magnitude.

The file http://www.ingber.com/asa_papers has references to the the use of ASA by some other researchers, e.g., in studies ranging from: comparisons among SA algorithms and between ASA and genetic algorithms, tabu and hillclimbing [51-55], to molecular models [56], to imaging [57], to neural networks [58], to econometrics [59], to geophysical inversion [60], to wide-spread use in financial institutions [48], etc.

\subsection{Description of Fits}

\subsection{1. $x$-Indicator of Market Contexts}

Our studies of contexts of markets well recognized by option traders to have significantly different volatility behavior show that the exponents $x$ are reasonably faithful indicators defining these different contexts. 
We feel the two-factor model is more accurate because the data indeed demonstrate stochastic volatility [4]. We also note that the two-factor $x$ 's are quite robust and uniform when being fit by ASA across the last few years; this is not true of the one-factor ASA fitted $x$ 's. Differences are primarily due to the stochastic $\sigma$ parameters, in accord with the sense traders have about the nature of changing volatilities across this time period. When stochastic volatility is properly treated, it is interesting that the two-factor $x$ 's are not so far from the BS $x=1$.

Fig. 3 shows the one-factor and two-factor exponents, the two-factor correlation $\rho$, and the raw data used for these fits, the ED contract expiring in September 1999 during the period June 1998 through March 1999, using a moving two-month window of data. In ASA, we placed boundaries on the onefactor exponent to lie between -3 and 2, just because we did not see any reason to examine models with very extreme $x$ 's.

The one-factor $x$ 's, albeit they are stochastic, do exhibit systematics of market contexts. For example, in our 1998 fits, there are two distinct periods, positive $x$ until the last quarter and then negative $x$. Once the fits reach 1999, $x$ turns positive again.

Fig. 3.

It should be noted that our two-factor fits use price and implied volatility data. Of course, the implied volatility data is derived by vendors from standard Black-Scholes-type calculations, whereas we are fitting a multivariate $S^{x}$ process. In practice, over the range of the data to be fit, the basis-point volatility in the empirical data, independent of any options model, is approximately the same for any $x$ we are considering. Also, other parameters in the model are involved in the fitting process, e.g., especially $v$ and $\varepsilon$ in the $\sigma$ equation, which offer tight resolution of the model within reasonable variances.

Fig. 4 shows the mean and standard deviation of the short-time differenced one-factor exponents. The one-factor exponents exhibit a random process that is approximately defined as a simple normal process $\eta_{x}$ with mean $\mu_{x}$ and standard deviation $\sigma_{x}$,

$$
\dot{x}=\frac{d x}{d t}=\mu_{x}+\sigma_{x} \eta_{x}
$$

When averaging over a several month period, we can derive $\mu_{x} \approx 0$ and have $\sigma_{x}$ essentially span all $x$ 's. 
However, it is clear that there are shorter periods of stochastic $x$ which can be modeled independently, yielding a one-factor $x$ as an indicator of market contexts. An additional problem arises if too short periods are selected for moving averages; drift can become significant for large movement in the price data. This is only a practical problem, in that traders most often compare volatilities across models over time periods which are large enough so that drifts are negligible.

Most important, the reasonable interpretation of our results is that suppression of stochastic volatility in the one-factor model just leaks out into stochasticity of parameters in the model, e.g., especially in $x$. By comparison, the $x$-exponents in the two-factor fits are quite stable.

Fig. 4.

\section{PATH-INTEGRAL (PATHINT) DEVELOPMENT}

\subsection{PATHINT Outline}

The fits described above clearly demonstrate the need to incorporate stochastic volatility in option pricing models. If one-factor fits are desired, e.g., for efficiency of calculation, then at the least the exponent of price $x$ should be permitted to freely adapt to the data. In either case, it is required to develop a full set of Greeks for trading. To meet these needs, we have used a path-integral code, PATHINT, described below, with great success. At this time, the two-factor code takes too long to run for daily use, but it proves to be a good weekly baseline for the one-factor code.

The PATHINT algorithm develops the long-time probability distribution from the Lagrangian fit by the first optimization code. A robust and accurate histogram-based (non-Monte Carlo) path-integral algorithm to calculate the long-time probability distribution has been developed to handle nonlinear Lagrangians [17-19,21,61-63],

The histogram procedure recognizes that the distribution can be numerically approximated to a high degree of accuracy as sum of rectangles at points $M_{i}$ of height $P_{i}$ and width $\Delta M_{i}$. For convenience, just consider a one-dimensional system. The above path-integral representation can be rewritten, for each of its intermediate integrals, as 


$$
\begin{aligned}
& P(M ; t+\Delta t)=\int d M^{\prime}\left[g_{s}^{1 / 2}(2 \pi \Delta t)^{-1 / 2} \exp \left(-L_{s} \Delta t\right)\right] P\left(M^{\prime} ; t\right)=\int d M^{\prime} G\left(M, M^{\prime} ; \Delta t\right) P\left(M^{\prime} ; t\right) \\
& P(M ; t)=\sum_{i=1}^{N} \pi\left(M-M_{i}\right) P_{i}(t) \\
& \pi\left(M-M_{i}\right)=\left\{\begin{array}{l}
0,\left(M_{i}-\frac{1}{2} \Delta M_{i-1}\right) \leq M \leq\left(M_{i}+\frac{1}{2} \Delta M_{i}\right), \\
1, \text { otherwise },
\end{array}\right.
\end{aligned}
$$

which yields

$$
\begin{aligned}
& P_{i}(t+\Delta t)=T_{i j}(\Delta t) P_{j}(t) \\
& T_{i j}(\Delta t)=\frac{2}{\Delta M_{i-1}+\Delta M_{i}} \int_{M_{i}-\Delta M_{i-1} / 2}^{M_{i}+\Delta M_{i} / 2} d M \int_{M_{j}-\Delta M_{j-1} / 2}^{M_{j}+\Delta M_{j} / 2} d M^{\prime} G\left(M, M^{\prime} ; \Delta t\right) .
\end{aligned}
$$

$T_{i j}$ is a banded matrix representing the Gaussian nature of the short-time probability centered about the (varying) drift.

Care must be used in developing the mesh in $\Delta M^{G}$, which is strongly dependent on the diagonal elements of the diffusion matrix, e.g.,

$$
\Delta M^{G} \approx\left(\Delta t g^{|G||G|}\right)^{1 / 2} .
$$

Presently, this constrains the dependence of the covariance of each variable to be a nonlinear function of that variable, albeit arbitrarily nonlinear, in order to present a straightforward rectangular underlying mesh. Below we address how we have handled this problem in our two-factor stochastic-volatility model.

Fitting data with the short-time probability distribution, effectively using an integral over this epoch, permits the use of coarser meshes than the corresponding stochastic differential equation. The coarser resolution is appropriate, typically required, for numerical solution of the time-dependent pathintegral: By considering the contributions to the first and second moments of $\Delta M^{G}$ for small time slices $\theta$, conditions on the time and variable meshes can be derived [61]. The time slice essentially is determined by $\theta \leq \bar{L}^{-1}$, where $\bar{L}$ is the "static" Lagrangian with $d M^{G} / d t=0$, throughout the ranges of $M^{G}$ giving the most important contributions to the probability distribution $P$. The variable mesh, a function of $M^{G}$, is optimally chosen such that $\Delta M^{G}$ is measured by the covariance $g^{G G^{\prime}}$, or $\Delta M^{G} \sim\left(g^{G G} \theta\right)^{1 / 2}$. 
The PATHINT algorithm in its present form can "theoretically" handle any n-factor model subject to its diffusion-mesh constraints. In practice, the calculation of 3-factor models likely will wait until gigahertz speeds and giga-byte RAM are commonplace.

\subsection{Development of Long-Time Probabilities}

The noise determined empirically as the diffusion of the data is the same independent of $x$ within our approach. Therefore, setting the strike $X$ to the $S$ underlying, the at-the-money (ATM) diffusions, the square of the "basis-point volatilities" (BPV), are scaled to be equivalent. Then, there is not a very drastic change in ATM option prices for different exponents $x$. This is not the case for out of the money strikes. This implies that current pricing models are not radically mispricing the markets, but there still are significant changes in Greeks using more sophisticated models.

\subsection{Dependence of Probabilities on $S$ and $x$}

Fig. 5 gives examples of the short-time distribution evolved out to $T=0.5$ year for $x$ in $\{-1,0,1$, $2\}$, with 500 intermediate epochs/foldings, and BS $\sigma=0.0075$. Each calculation scales $\sigma$ by multiplying by $S / F\left(S, S_{0}, S_{\infty}, x, y\right)$.

Fig. 5.

Fig. 6 gives an example of a two-factor distribution evolved out to $T=0.5$ year for $x=0.7$.

Fig. 6.

\subsection{Two-Factor Volatility and PATHINT Modifications}

In our two-factor model, the mesh of $S$ would depend on $\sigma$ and cause some problems in any PATHINT grid to be developed in $S-\sigma$.

For some time we have considered how to handle this generic problem for $n$-factor multivariate systems with truly multivariate diffusions. In one case, we have taken advantage of the Riemannian 
invariance of the probability distribution as discussed above, to transform to a system where the diffusions have only "diagonal" multiplicative dependence [18]. However, this leads to cumbersome numerical problems with the transformed boundary conditions [19]. Another method, not yet fully tested, is to develop a tiling of diagonal meshes for each factor $i$ that often are suitable for off-diagonal regions in an $n$-factor system, e.g.,

$$
\begin{aligned}
& \Delta M_{k}^{i}=2^{m_{k}^{i}} \Delta M_{0}^{i} \\
& \Delta M_{0}^{i} \approx \sqrt{g_{k_{0}}^{|i||i|} \Delta t}
\end{aligned}
$$

where the mesh of variable $i$ at a given point labeled by $k$ is an exponentiation of 2 , labeled by $m_{k}^{i}$; the

integral power $m_{k}^{i}$ is determined such that it gives a good approximation to the diagonal mesh given by the one-factor PATHINT mesh conditions, in terms of some minimal mesh $\Delta M_{0}^{i}$, throughout regions of the Lagrangian giving most important contributions to the distribution as predetermined by a scan of the system. This tiling of the kernel is to be used together with interpolation of intermediate distributions.

The results of our study here are that, after the at-the-money BPV are scaled to be equivalent, there is not a very drastic change in the one-factor ATM Greeks developed below. Therefore, while we have not at all changed the functional dependence of the Lagrangian on $S$ and $\sigma$, we have determined our meshes using a diffusion for the $S$ equation as $\sigma_{0} F\left(S, S_{0}, S_{\infty}, x, y\right)$, where $\sigma_{0}$ is determined by the same BPV-equivalent condition as imposed on the one-factor models. This seems to work very well, especially since we have taken our $\sigma$ equation to be normal with a limited range of influence in the calculations. Future work yet has to establish a more definitive distribution for $\sigma$.

\section{CALCULATION OF DERIVATIVES}

\subsection{Primary Use of Probabilities For European Options}

We could have modified PATHINT to develop the distribution of the option value back in time from expiration. This is the standard approach used by CRR, explicit and implicit Crank-Nicolson models, etc [28].

However, we decided to take advantage of the accuracy of PATHINT enhanced by normalizing the distribution as well as the kernel at each iteration. Therefore, we have calculated our option prices and 
Greeks using the most elementary and intuitive definition of the option's price $V$ [64], which is the expected value

$$
V=<\max [z(S-X), 0]>, \begin{cases}z=1, & \text { call } \\ z=-1, & \text { put }\end{cases}
$$

where $X$ is the strike price, and the expected value $\langle$.$\rangle is taken with respect to the risk-neutral$ distribution of the underlying market $S$. It should be noted that, while the standard approach of developing the option price delivers at the present time a range of underlying values for a given strike, our approach delivers a more practical complete range of strikes for a given underlying at the present time. The risk-neutral distribution is effectively calculated taking the drift as the cost-of-carry $b$ times $S$, using the above arguments leading to the BS formula. We have designed our codes to use parameters risk-freerate $r$ and cost-of-carry $b$ such that

$$
\begin{aligned}
& b=r, \text { cost of carry on nondividend stock } \\
& b=r-q, \text { cost of carry on stock paying dividend yield } q \\
& b=0, \text { cost of carry on future contract } \\
& b=r-r_{f}, \text { cost of carry on currency with rate } r_{f}
\end{aligned}
$$

which is similar to how generalized European BS codes use $b$ and $r$ [65].

Using this approach, the European price $V_{E}$ is calculated as

$$
V_{E}=\left\langle\max \left[z\left(e^{(b-r) T} S_{T}-e^{-r T} X\right), 0\right]>\right.
$$

The American price $V_{A}$ must be calculated using a different kernel going back in time from expiration, using as "initial conditions" the option values used in the above average. This kernel is the transposed matrix used for the European case, and includes additional drift and "potential" terms due to the need to develop this back in time. This can be viewed as requiring the adjoint partial differential equation or a postpoint Lagrangian in real time. The Greeks $\{\Delta, \Gamma, \Theta\}$ are directly taken off the final developed option. We get excellent results for all Greeks. 


\subsection{PATHINT Baselined to CRR and BS}

The CRR method is a simple binomial tree which in a specific limit approaches the BS partial differential equation. It has the virtues of being fast and readily accommodates European and American calculations. However, it suffers a number of numerical problems, e.g., a systematic bias in the tree approximation and an oscillatory error as a function of the number of intermediate epochs/iterations in its time mesh. Some Greeks like $\{\Delta, \Gamma, \Theta\}$ can be directly taken off the tree used for pricing with reasonable approximations (at epochs just before the actual current time). The first problem for American options can be alleviated somewhat by using the variant method [5],

$$
\mathrm{CRR}_{\text {variant }}=\mathrm{CRR}_{\text {American }}-\mathrm{CRR}_{\text {European }}+\mathrm{BS}
$$

The second problem can be alleviated somewhat by averaging runs of $n$ iterations with runs of $n+1$ iterations [66]. This four-fold increase of runs is rarely used, though perhaps it should be more often. Furthermore, if increased accuracy in price is needed in order to take numerical derivatives, typically 200-300 iterations should be used for expirations some months away, not 30-70 as too often used in practice.

When taking numerical derivatives there can arise a need to tune increments taken for the differentials. For some Greeks like $\Delta$ and $\Gamma$ the size of the best differentials to use may vary with strikes that have different sensitivities to parts of the underlying distribution. One method of building in some adaptive flexibility across many such strikes is to increase the order of terms used in taking numerical derivatives. (This was not required for results presented here.) For example, it is straightforward to verify that, while the central difference

$$
\frac{d f}{d x}=\frac{f(x+d x)-f(x-d x)}{2 d x}
$$

is typically good to $o\left((d x)^{3}\right)$,

$$
\frac{d f}{d x}=\frac{-f(x+2 d x)+8 f(x+d x)-8 f(x-d x)+f(x-2 d x)}{12 d x}
$$

is typically good to $o\left((d x)^{5}\right)$. Similarly, while

$$
\frac{d^{2} f}{d x^{2}}=\frac{f(x+d x)-2 f(x)+f(x-d x)}{d x d x}
$$

is typically good to $\left((d x)^{4}\right)$, 


$$
\frac{d^{2} f}{d x^{2}}=\frac{-d(x+2 d x)+16 f(x+d x)-30 f(x)+16 f(x-d x)-f(x-2 d x)}{12 d x d x}
$$

is typically good to $\left((d x)^{6}\right)$.

Table 1 gives an example of baselining our one-factor PATHINT code to the CRR and BS codes using the above safeguards for an option whose American price is the same as its European counterpart, a typical circumstance [65]. In the literature, the CRR code is most often taken as the benchmark for American calculations. We took the number of intermediate epochs/points to be 300 for each calculation. Parameters used for this particular ATM call are $T=0.5$ years, $r=0.05, b=0, \sigma=0.10$.

\section{Table 1.}

Tests with American CRR and American PATHINT lead to results with the same degrees of accuracy.

\subsection{Two-Factor PATHINT Baselined to One-Factor PATHINT}

Previous papers and tests have demonstrated that the two-factor PATHINT code performs as expected. The code was developed with only a few lines to be changed for running any $n$-factor problem. Tests were performed by combining two one-factor problems, and there is no loss of accuracy. However, here we are making some additional mesh approximations as discussed above to accommodate $\sigma$ in the $S$ diffusion. This seems quite reasonable, but there is no sure test of the accuracy. We indeed see that the ATM results are very close across $x$ 's, similar to our ATM comparisons between BS and our one-factor PATHINT results for various $x$ 's, where again scaling is performed to have all models used the same BPV (using the $\sigma_{0}$ procedure described above for the two-factor model).

The logical extension of Greeks for the two-factor model is to develop derivatives of price with respect to $\rho$ and $\varepsilon$ in $\sigma$ volatility equation. However, we did not find a two-factor proxy for the one-factor vega, the derivative of price with respect to the one-factor $\sigma$ constant. We get very good ATM vega comparisons between BS and our one-factor models with various $x$ 's. We tried simply multiplying the noise in the two-factor stochastic volatility in the price equation by a parameter with deviations from 1 to get numerical derivatives of PATHINT solutions, but this did not give good agreement with the ATM 
BPV-scaled BS vega. Perhaps this is not too surprising, especially given the correlation substantial $\rho$ between the price and volatility equations which we do not neglect.

\section{CONCLUSIONS}

The results of our study are that, after the at-the-money basis-point volatilities are scaled to be equivalent, there is only a very small change in option prices for different exponents $x$, both for the onefactor and two-factor models. There still are significant differences in Greeks using more sophisticated models, especially for out-of-the-money options. This implies that current pricing models are not radically mispricing the markets.

Our studies point to contexts of markets well recognized by option traders to have significantly different volatility behavior. Suppression of stochastic volatility in the one-factor model just leaks out into stochasticity of parameters in the model, e.g., especially in $x$. Our studies show that the two-factor exponents $x$ are reasonably faithful indicators defining these different contexts. By comparison, the $x$ exponents in the two-factor fits are quite stable. As such, especially the two-factor $x$ can be considered as a "context indicator" over a longer time scale than other indicators typically used by traders.

The two-factor fits also exhibit differences due to the $\sigma$ parameters, including the $\rho$ correlations, in accord with the sense traders have about the nature of changing volatilities across this time period. When stochastic volatility is properly treated, the two-factor $x$ 's also are quite close to the BS $x=1$.

Modern methods of developing multivariate nonlinear multiplicative Gaussian-Markovian systems are quite important, as there are many such systems and the mathematics must be diligently exercised if such models are to faithfully represent the data they describe. Similarly, sophisticated numerical techniques, e.g., global optimization and path integration are important tools to carry out the modeling and fitting to data without compromising the model, e.g., by unwarranted quasi-linear approximations. Three quite different systems have benefited from this approach.

The large-scale modeling of neocortical interactions has benefited from the use of intuitive constructs that yet are faithful to the complex algebra describing this multiple-scaled complex system. For example, canonical-momenta indicators have been successfully applied to multivariate financial markets. 
It is clear that ASA optimization and PATHINT path-integral tools are very useful to develop the algebra of statistical mechanics for a large class of nonlinear stochastic systems encountered in finance. However, it also is clear that each system typically presents its own non-typical unique character and this must be included in any such analysis. A virtue of this statistical mechanics approach and these associated tools is they appear to be flexible and robust to handle quite different systems.

\section{ACKNOWLEDGMENTS}

We thank Donald Wilson for his support and discussions on trading Eurodollar options, Man Wei Tam for his work in preparing the data and for discussions throughout the course of this project, and Yuri Pinelis for several discussions on our modelling approach. Implied volatility and yield data was extracted from the MIM database of Logical Information Machines (LIM). 


\section{REFERENCES}

1. L. Ederington and W. Guan, Is implied volatility an informationally efficient and effective predictor of future volatility?, U Oklahoma, Norman, OK, (1998).

2. G. Bakshi, C. Cao, and Z. Chen, Pricing and hedging long-term options, Pennsylvania State U, University Park, PA, (1998).

3. L. Ingber, Some Applications of Statistical Mechanics of Financial Markets, LIR-98-1-SASMFM, Lester Ingber Research, Chicago, IL, (1998).

4. L. Ingber and J.K. Wilson, Volatility of volatility of financial markets, Mathl. Computer Modelling 29 (5), 39-57 (1999).

5. J.C. Hull, Options, Futures, and Other Derivatives, Third Edition, Prentice Hall, Upper Saddle River, NJ, (1997).

6. J.C. Jackwerth, Recovering risk aversion from option prices and realized returns, London Business School, London, UK, (1998).

7. F.A. Longstaff and E.S. Schwartz, Valuing American options by simulation: A simple least-squares approach, Capital Management Sciences, Los Angels, CA, (1998).

8. L. Ingber, Statistical mechanics of nonlinear nonequilibrium financial markets, Math. Modelling 5 (6), 343-361 (1984).

9. L. Ingber, Statistical mechanical aids to calculating term structure models, Phys. Rev. A 42 (12), 7057-7064 (1990).

10. L. Ingber, M.F. Wehner, G.M. Jabbour, and T.M. Barnhill, Application of statistical mechanics methodology to term-structure bond-pricing models, Mathl. Comput. Modelling 15 (11), 77-98 (1991).

11. L. Ingber, Statistical mechanics of nonlinear nonequilibrium financial markets: Applications to optimized trading, Mathl. Computer Modelling 23 (7), 101-121 (1996).

12. L. Ingber, Canonical momenta indicators of financial markets and neocortical EEG, in Progress in Neural Information Processing, (Edited by S.-I. Amari, L. Xu, I. King, and K.-S. Leung), pp. 777-784, Springer, New York, (1996). 
13. C. Azariadis, Self-fulfilling prophecies, J. Econ. Theory 25, 380-396 (1981).

14. L. Ingber, Adaptive Simulated Annealing (ASA), Global optimization C-code, Caltech Alumni Association, Pasadena, CA, (1993).

15. J. C. Cox, S. A. Ross, and M. Rubenstein, Option pricing: A simplified approach, J. Fin. Econ. 7, 229-263 (1979).

16. L. Ingber, Data mining and knowledge discovery via statistical mechanics in nonlinear stochastic systems, Mathl. Computer Modelling 27 (3), 9-31 (1998).

17. L. Ingber, H. Fujio, and M.F. Wehner, Mathematical comparison of combat computer models to exercise data, Mathl. Comput. Modelling 15 (1), 65-90 (1991).

18. L. Ingber, Statistical mechanics of neocortical interactions: Path-integral evolution of short-term memory, Phys. Rev. E 49 (5B), 4652-4664 (1994).

19. L. Ingber and P.L. Nunez, Statistical mechanics of neocortical interactions: High resolution pathintegral calculation of short-term memory, Phys. Rev. E 51 (5), 5074-5083 (1995).

20. L. Ingber, R. Srinivasan, and P.L. Nunez, Path-integral evolution of chaos embedded in noise: Duffing neocortical analog, Mathl. Computer Modelling 23 (3), 43-53 (1996).

21. L. Ingber, Path-integral evolution of multivariate systems with moderate noise, Phys. Rev. E 51 (2), 1616-1619 (1995).

22. Federal Reserve Bank, Instruments of the Money Markets, Seventh Edition, Federal Reserve Bank of Richmond, Richmond, VA, (1993).

23. L. Bachelier, Théorie de la Spéculation, Annales de l'Ecole Normale Supérieure 17, 21-86 (1900).

24. M. C. Jensen, Some anomalous evidence regarding market efficiency, an editorial introduction, $J$. Finan. Econ. 6, 95-101 (1978).

25. B. B. Mandelbrot, When can price be arbitraged efficiently? A limit to the validity of the random walk and martingale models, Rev. Econ. Statist. 53, 225-236 (1971).

26. S. J. Taylor, Tests of the random walk hypothesis against a price-trend hypothesis, J. Finan. Quant. Anal. 17, 37-61 (1982).

27. P. Brown, A. W. Kleidon, and T. A. Marsh, New evidence on the nature of size-related anomalies in stock prices, J. Fin. Econ. 12, 33-56 (1983). 
28. P. Wilmott, S. Howison, and J. Dewynne, The Mathematics of Financial Derivatives, Cambridge U Press, Cambridge, (1995).

29. F.J. Fabozzi, Treasury Securities and Derivatives, Fabozzi Associates, New Hope, PA, (1998).

30. L. Ingber, Statistical mechanics of neocortical interactions: A scaling paradigm applied to electroencephalography, Phys. Rev. A 44 (6), 4017-4060 (1991).

31. K.S. Cheng, Quantization of a general dynamical system by Feynman's path integration formulation, J. Math. Phys. 13, 1723-1726 (1972).

32. H. Dekker, Functional integration and the Onsager-Machlup Lagrangian for continuous Markov processes in Riemannian geometries, Phys. Rev. A 19, 2102-2111 (1979).

33. R. Graham, Path-integral methods in nonequilibrium thermodynamics and statistics, in Stochastic Processes in Nonequilibrium Systems, (Edited by L. Garrido, P. Seglar, and P.J. Shepherd), pp. 82-138, Springer, New York, NY, (1978).

34. F. Langouche, D. Roekaerts, and E. Tirapegui, Discretization problems of functional integrals in phase space, Phys. Rev. D 20, 419-432 (1979).

35. F. Langouche, D. Roekaerts, and E. Tirapegui, Short derivation of Feynman Lagrangian for general diffusion process, J. Phys. A 113, 449-452 (1980).

36. P. Hagedorn, Non-Linear Oscillations, Oxford Univ., New York, NY, (1981).

37. B. Oksendal, Stochastic Differential Equations, Springer, New York, NY, (1998).

38. J.M. Harrison and D. Kreps, Martingales and arbitrage in multiperiod securities markets, J. Econ. Theory 20, 381-408 (1979).

39. S.R. Pliska, Introduction to Mathematical Finance, Blackwell, Oxford, UK, (1997).

40. C.W. Gardiner, Handbook of Stochastic Methods for Physics, Chemistry and the Natural Sciences, Springer-Verlag, Berlin, Germany, (1983).

41. F. Langouche, D. Roekaerts, and E. Tirapegui, Functional Integration and Semiclassical Expansions, Reidel, Dordrecht, The Netherlands, (1982).

42. R. C. Merton, An intertemporal capital asset pricing model, Econometrica 41, 867-887 (1973). 
43. L. Ingber, Statistical mechanics of neocortical interactions: Stability and duration of the 7+-2 rule of short-term-memory capacity, Phys. Rev. A 31, 1183-1186 (1985).

44. L. Ingber, Very fast simulated re-annealing, Mathl. Comput. Modelling 12 (8), 967-973 (1989).

45. S. Kirkpatrick, C.D. Gelatt, Jr., and M.P. Vecchi, Optimization by simulated annealing, Science 220 (4598), 671-680 (1983).

46. S. Geman and D. Geman, Stochastic relaxation, Gibbs distribution and the Bayesian restoration in images, IEEE Trans. Patt. Anal. Mac. Int. 6 (6), 721-741 (1984).

47. H. Szu and R. Hartley, Fast simulated annealing, Phys. Lett. A 122 (3-4), 157-162 (1987).

48. M. Wofsey, Technology: Shortcut tests validity of complicated formulas, The Wall Street Journal 222 (60), B1 (1993).

49. L. Ingber, Simulated annealing: Practice versus theory, Mathl. Comput. Modelling 18 (11), 29-57 (1993).

50. L. Ingber, Adaptive simulated annealing (ASA): Lessons learned, Control and Cybernetics 25 (1), 33-54 (1996).

51. B. Rosen, Function optimization based on advanced simulated annealing, IEEE Workshop on Physics and Computation - PhysComp '92 , 289-293 (1992).

52. L. Ingber and B. Rosen, Genetic algorithms and very fast simulated reannealing: A comparison, Mathl. Comput. Modelling 16 (11), 87-100 (1992).

53. D.G. Mayer, J.A. Belward, and K. Burrage, Use of advanced techniques to optimize a multidimensional dairy model, Agricultural Systems 50, 239-253 (1996).

54. D.G. Mayer, J.A. Belward, K. Burrage, and M.A Stuart, Optimization of a dairy farm model Comparison of simulated annealing, simulated quenching and genetic algorithms, in Proceedings 1995 International Congress on Modelling and Simulation, 27-30 November 1995, University of Newcastle, pp. 33-38, (1995).

55. D.G. Mayer, P.M. Pepper, J.A. Belward, K. Burrage, and A.J. Swain, Simulated annealing - A robust optimization technique for fitting nonlinear regression models, in Proceedings 'Modelling, Simulation and Optimization' Conference, International Association of Science and Technology for Development (IASTED), 6-9 May 1996 Gold Coast, (1996). 
56. A. Su, S. Mager, S.L. Mayo, and H.A. Lester, A multi-substrate single-file model for ion-coupled transporters, Biophys. J 70, $762-777$ (1996).

57. K. Wu and M.D. Levine, 3-D shape approximation using parametric geons, Intl J Image and Vision Computing 15 (2), 143-158 (1997).

58. B. Cohen, Training synaptic delays in a recurrent neural network, M.S. Thesis, Tel-Aviv University, Tel-Aviv, Israel, (1994).

59. S. Sakata, High breakdown point estimation in econometrics, Ph.D. Thesis, University of California at San Diego, La Jolla, CA, (1995).

60. M.K. Sen and P.L. Stoffa, Global Optimization Methods in Geophysical Inversion, Elsevier, The NetherLands, (1995).

61. M.F. Wehner and W.G. Wolfer, Numerical evaluation of path-integral solutions to Fokker-Planck equations. I., Phys. Rev. A 27, 2663-2670 (1983).

62. M.F. Wehner and W.G. Wolfer, Numerical evaluation of path-integral solutions to Fokker-Planck equations. II. Restricted stochastic processes, Phys. Rev. A 28, 3003-3011 (1983).

63. M.F. Wehner and W.G. Wolfer, Numerical evaluation of path integral solutions to Fokker-Planck equations. III. Time and functionally dependent coefficients, Phys. Rev. A 35, 1795-1801 (1987).

64. L. Ingber, A simple options training model, Mathl. Computer Modelling 30 (5-6), 167-182 (1999).

65. E.G. Haug, The Complete Guide to Option Pricing Formulas, McGraw-Hill, New York, NY, (1997).

66. M. Broadie and J. Detemple, Recent advances in numerical methods for pricing derivative securities, in Numerical Methods in Finance, (Edited by L.C.G Rogers and D. Talay), pp. 43-66, Cambridge University Press, Cambridge, UK, (1997). 


\section{FIGURE CAPTIONS}

FIG. 1. (a) Comparison of Basis-Point Volatility (BPV), standard deviation of BPV (SDBPV), and standard deviation of differenced BPV (SDDBPV), for Front and Back contracts. (b) Comparison of standard deviation of differenced Basis-Point Volatility (SDDBPV) with and standard deviation of differenced Black-Scholes Volatility (SDDBSV), for Front and Back contracts. The SDDBSV have been scaled to the SDDBPV by multiplying them by the rounded average of the yields, i.e., 6.0. (c) BasisPoint Implied Volatility (BPIV) of Front and Back contracts. (d) Standard deviation of differenced BasisPoint Implied Volatility (SDDBPIV) of Front and Back contracts.

FIG. 2. (a) $F\left(S, S_{0}, S_{\infty}, x, y\right)$ for $x=1$, the Black-Scholes case. The other parameters are $S=5$, $S_{0}=0.5, S_{\infty}=20, y=0$. (b) $F\left(S, S_{0}, S_{\infty}, x, y\right)$ for $x=0$, the normal distribution. (c) $F\left(S, S_{0}, S_{\infty}, x, y\right)$ for $x=-1$. (d) $F\left(S, S_{0}, S_{\infty}, x, y\right)$ for $x=2$.

FIG. 3. (a) Weekly two-month moving-averaged one-factor and two-factor exponents for ED contract expiring in September 1999 during the period June 1998 through March 1999. (b) Weekly twomonth moving-averaged two-factor correlation $\rho$ for this same data. (c) Raw price data used in fits for the above parameters. (d) Implied-volatility data used in fits for the above parameters.

FIG. 4. The means and standard deviations of the short-time differenced one-factor exponents.

FIG. 5. The short-time probability distribution at time $T=0.5$ years for $x=1$, the (truncated) Black-Scholes distribution. The short-time probability distribution at time $T=0.5$ years for $x=0$, the normal distribution. The short-time probability distribution at time $T=0.5$ years for $x=-1$. The shorttime probability distribution at time $T=0.5$ years for $x=2$.

FIG. 6. A two-factor distribution evolved out to $T=0.5$ year for $x=0.7$. 


\section{TABLE CAPTIONS}

Table 1. Calculation of prices and Greeks are given for closed form BS (only valid for European options), binomial tree $\mathrm{CRR}_{\text {European }}, \mathrm{CRR}_{\text {American }}, \mathrm{CRR}_{\text {variant }}$, and PATHINT. As verified by calculation, the American option would not be exercised early, so the PATHINT results are identical to the European option. The $\mathrm{CRR}_{\text {American }}$ differs somewhat from the $\mathrm{CRR}_{\text {European }}$ due to the discrete nature of the calculation. All CRR calculations include averaging over 300 and 301 iterations to minimize oscillatory errors. 
(a) Historical BPV

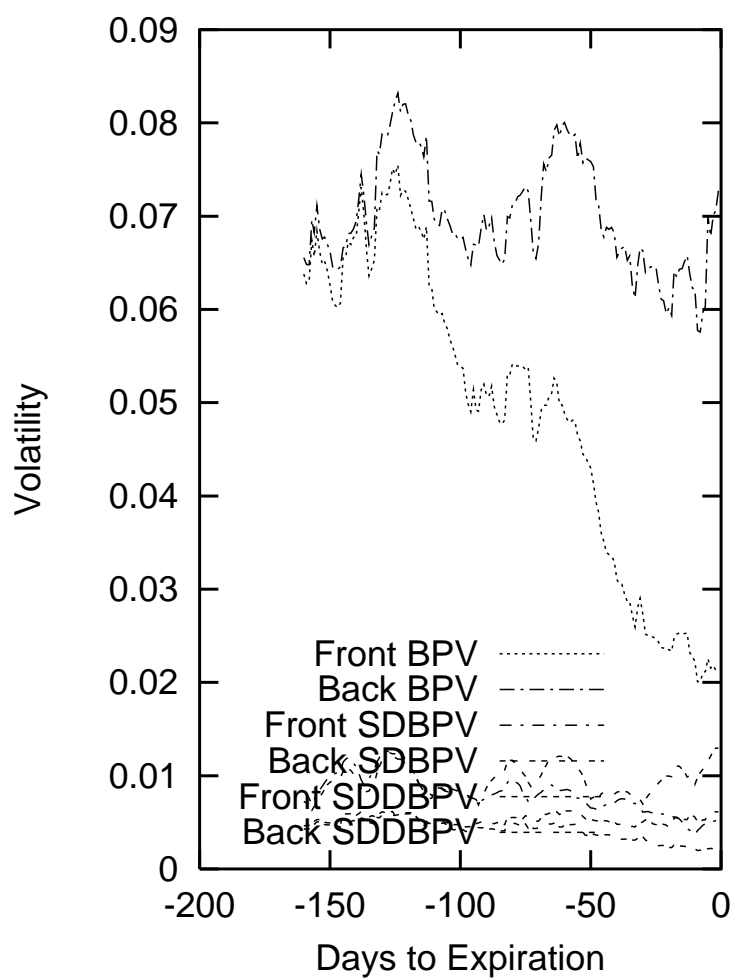

(c) Implied BPV

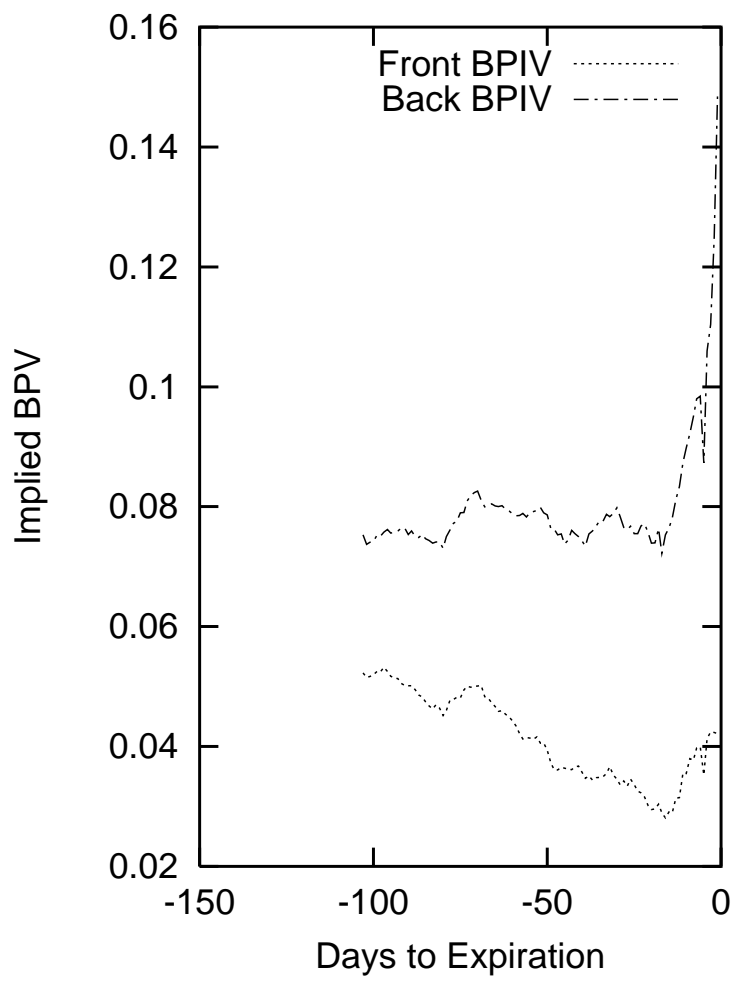

(b) Historical Differenced BPV

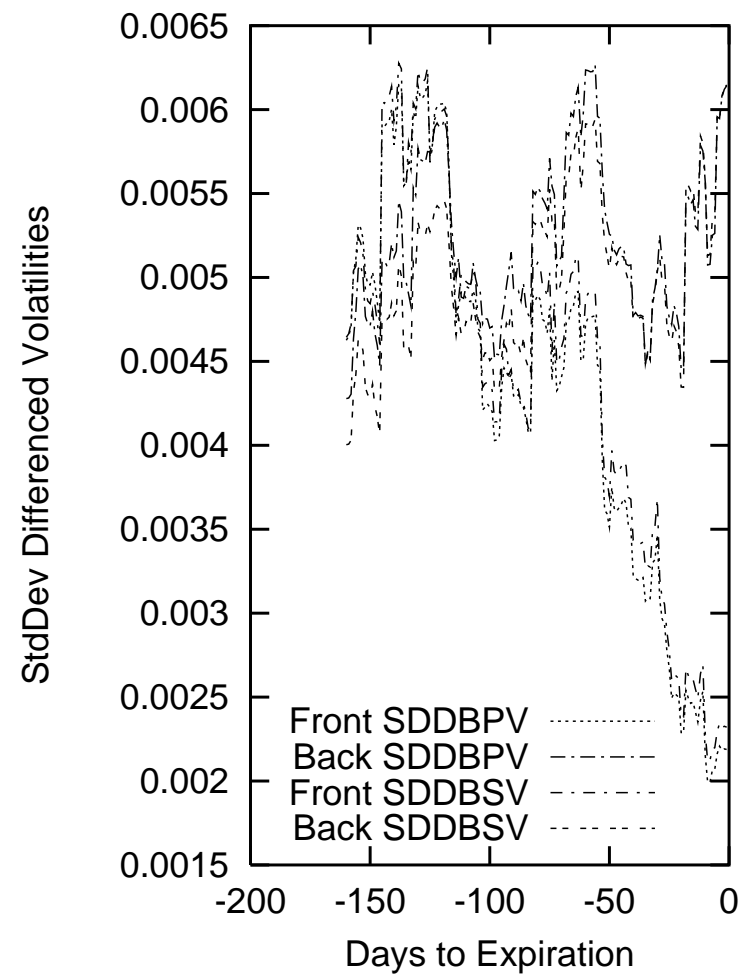

(d) Implied Differenced BPV

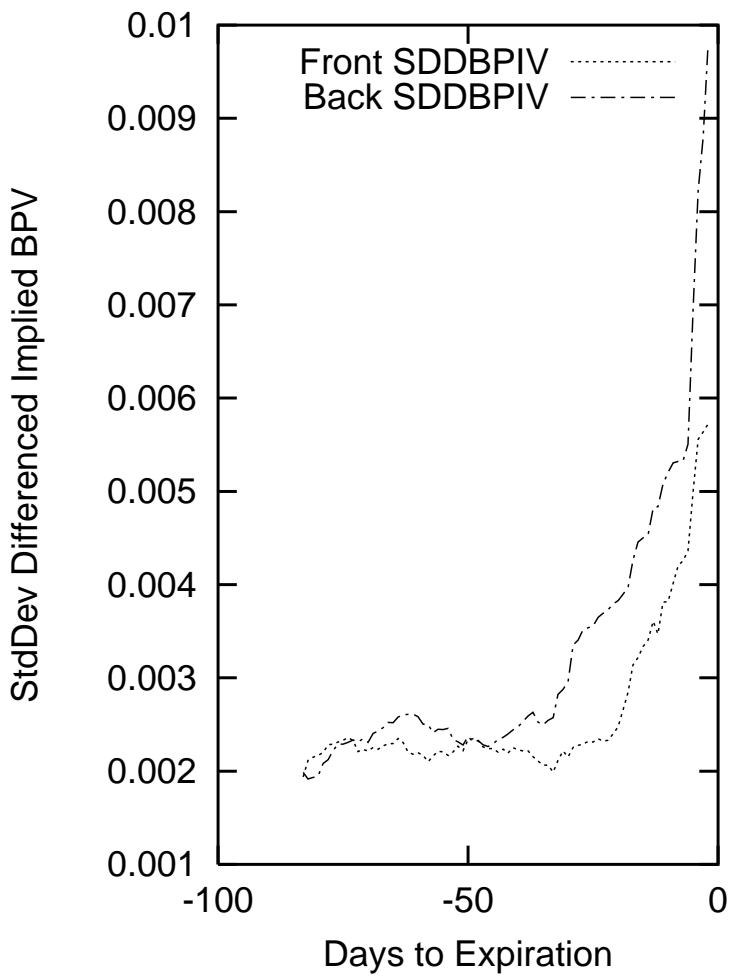


(a) $x=1$

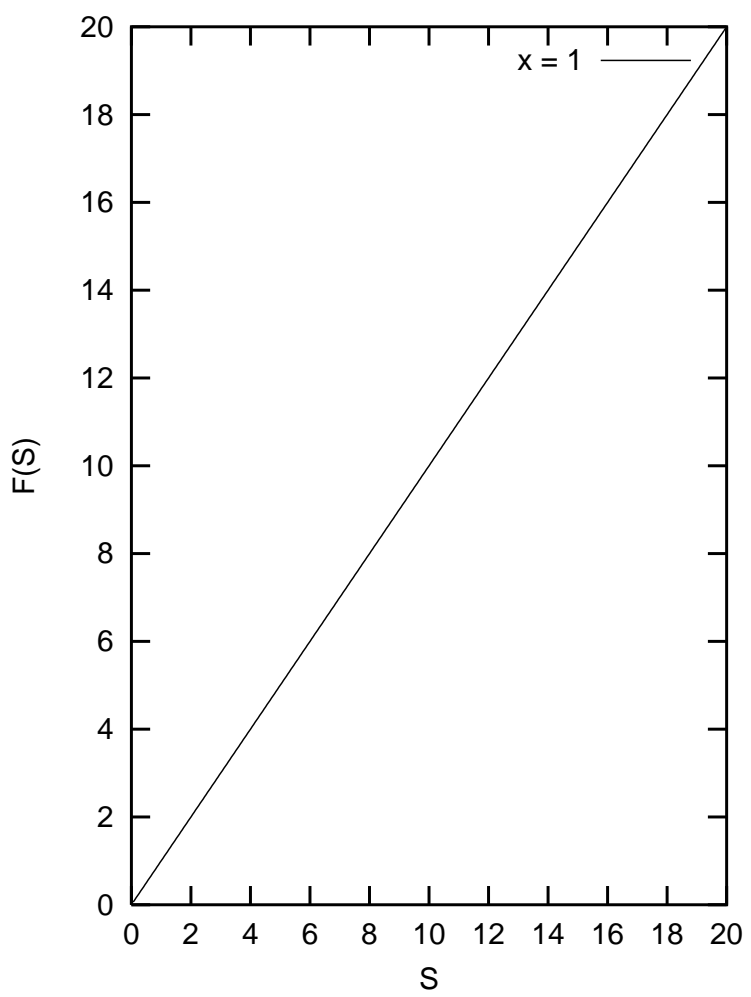

(c) $x=-1$

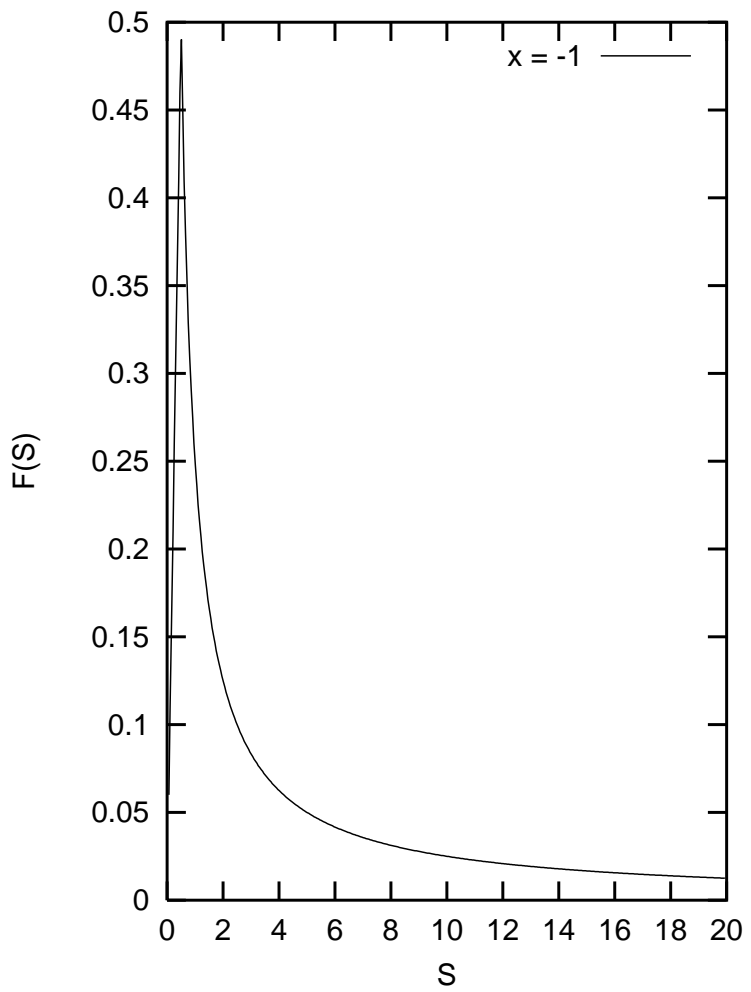

(b) $x=0$

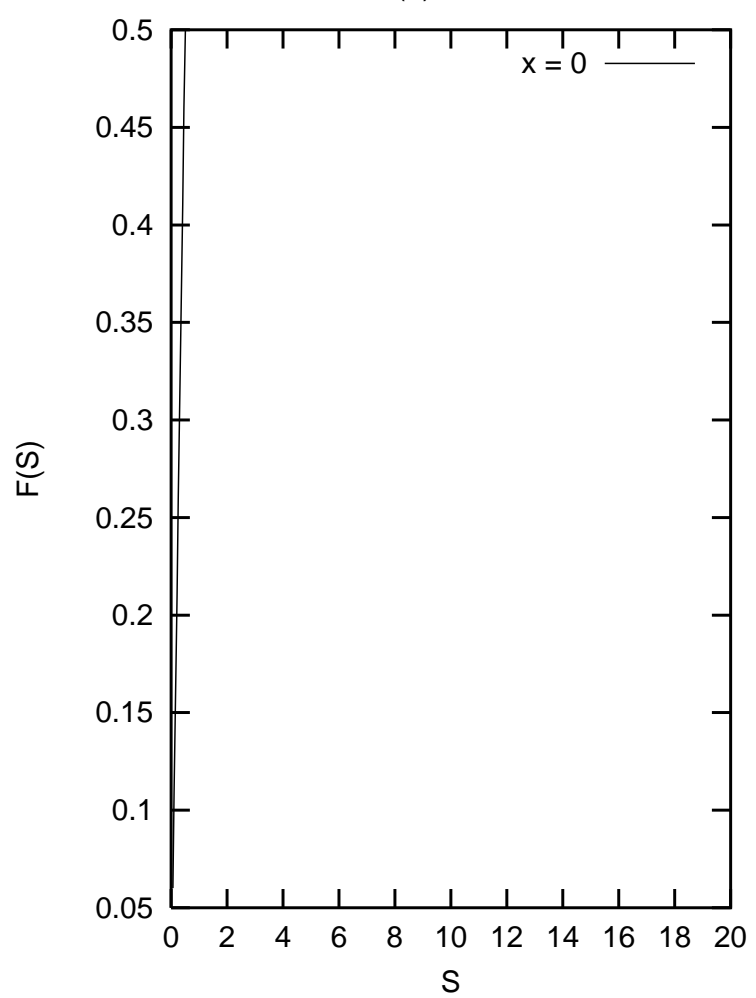

(d) $x=2$

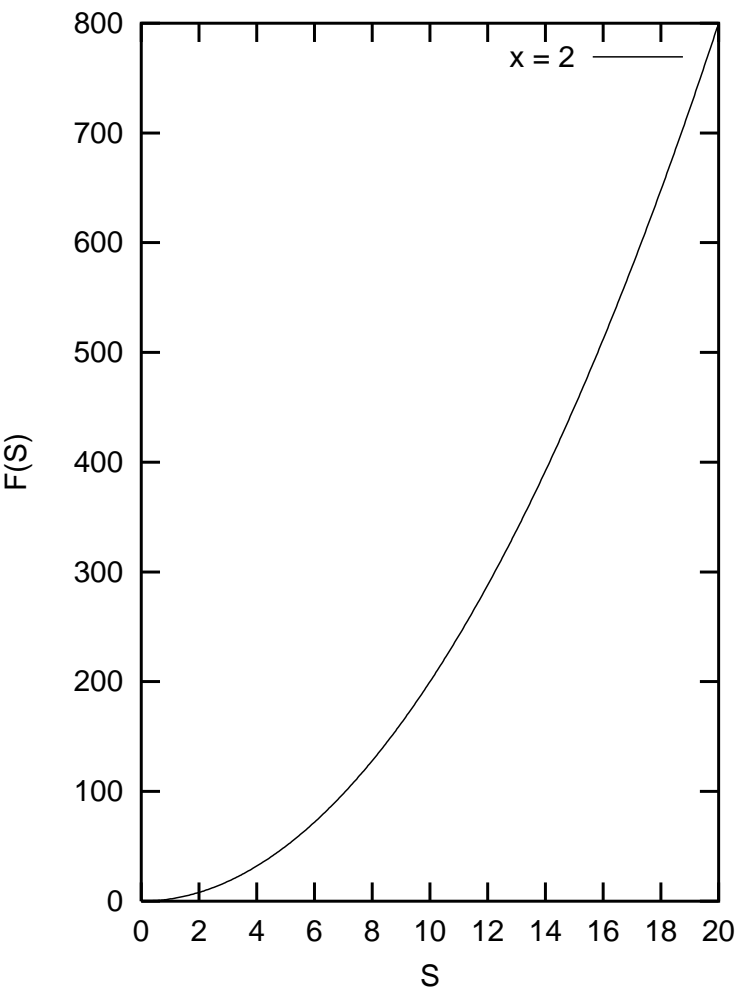


(a) Exponent

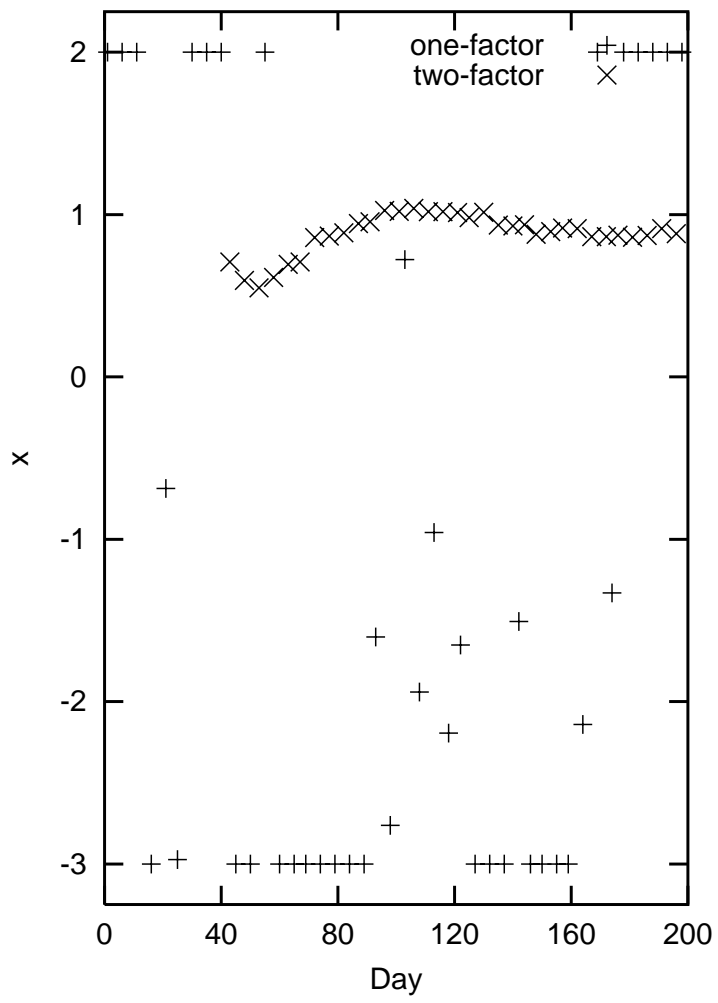

(c) ED Price

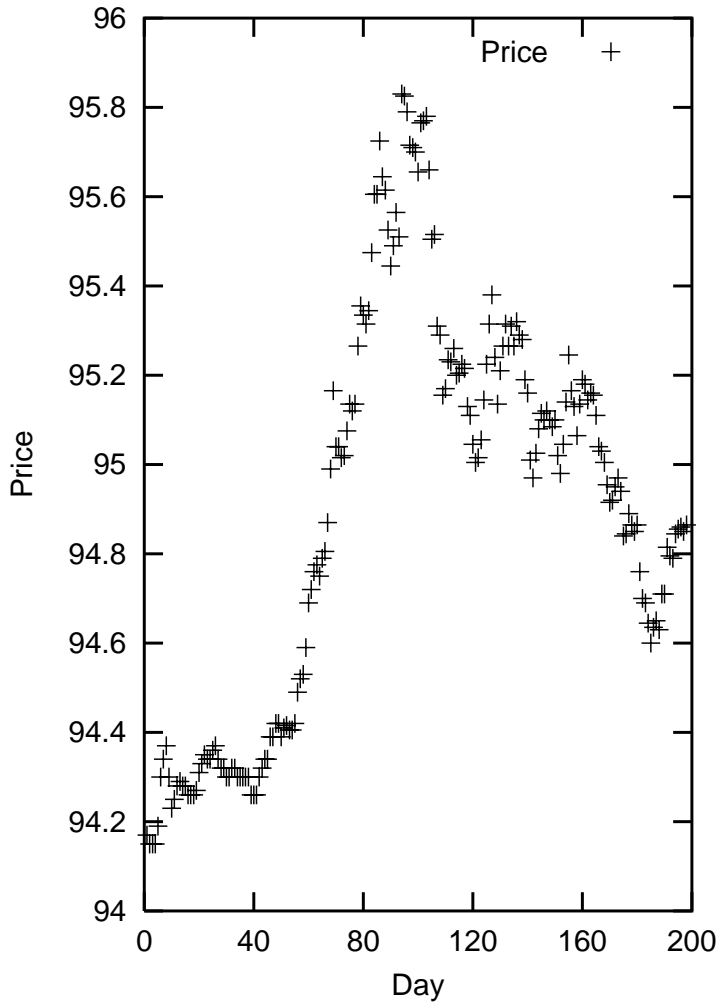

(b) Correlation

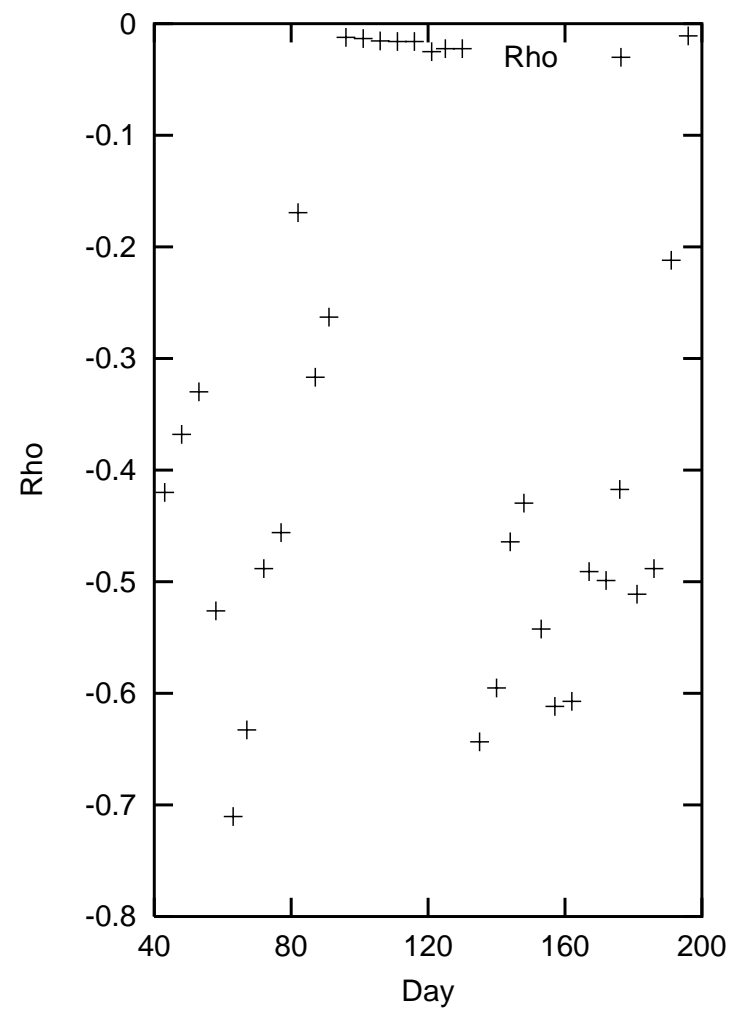

(d) ED Implied Volatility

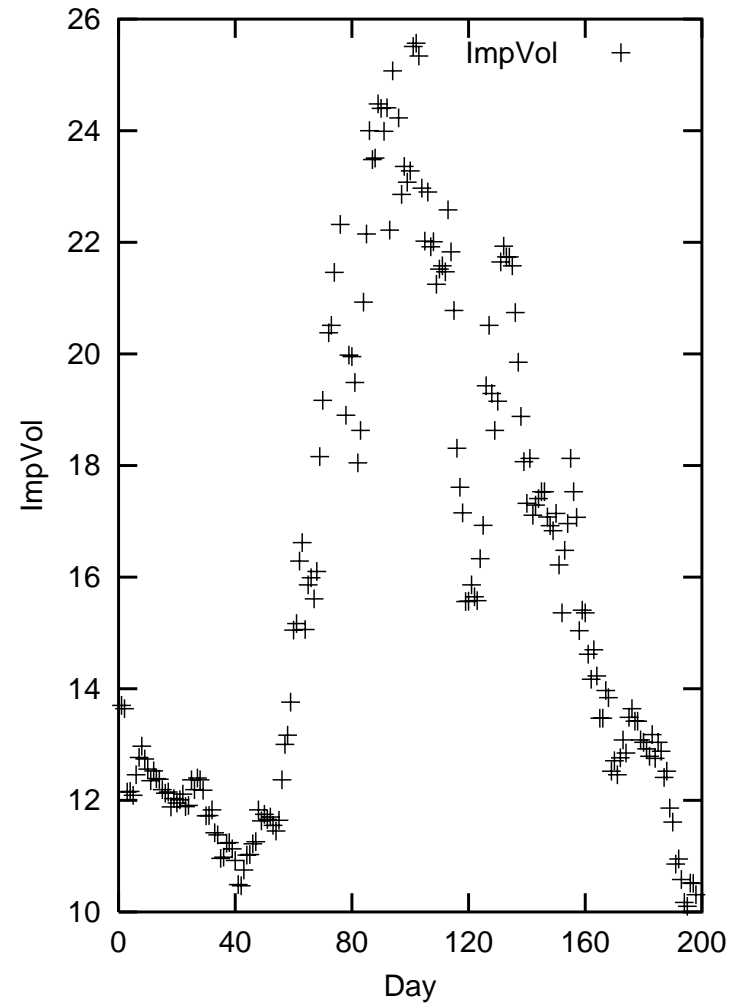




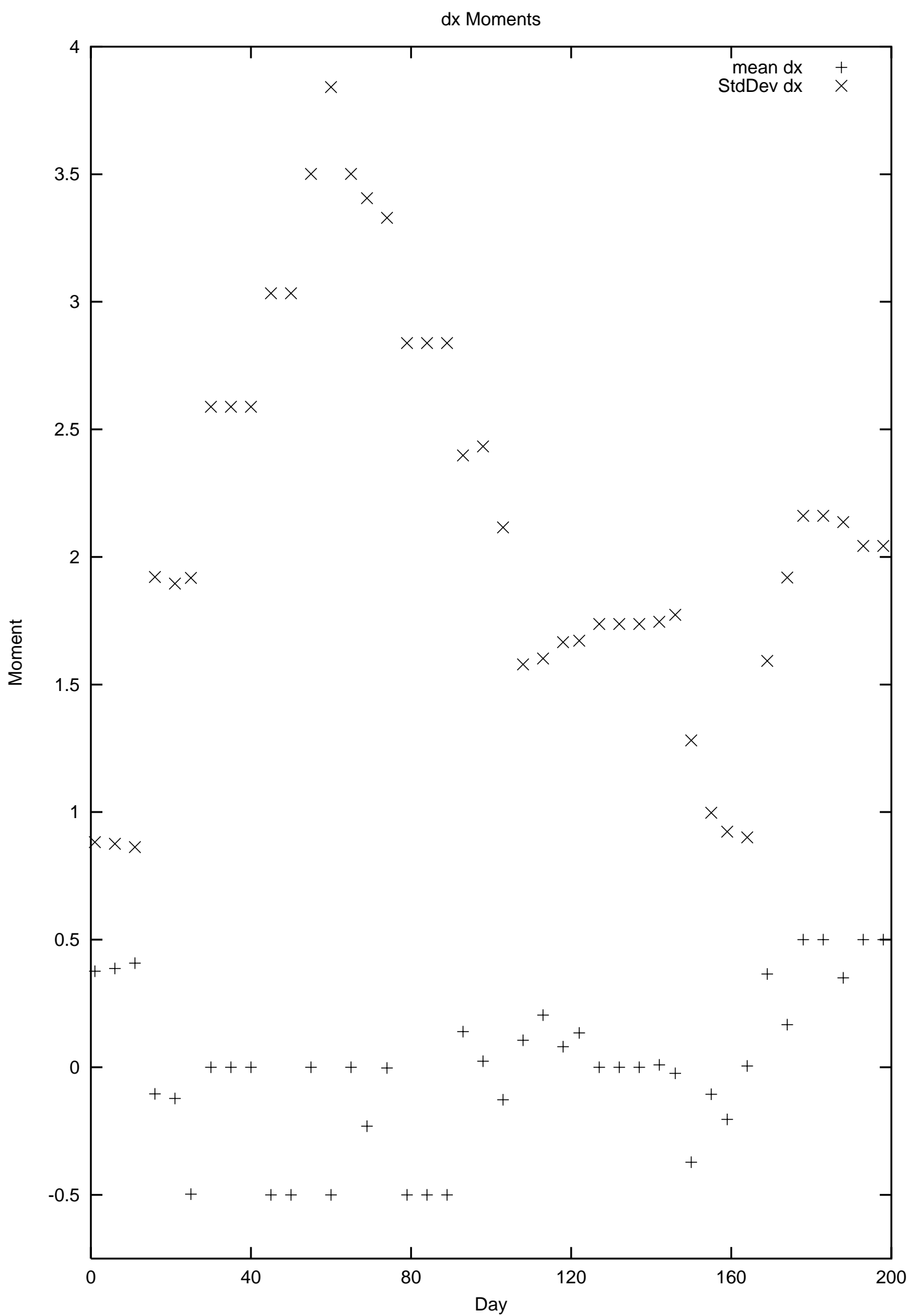


(a) $x=1$

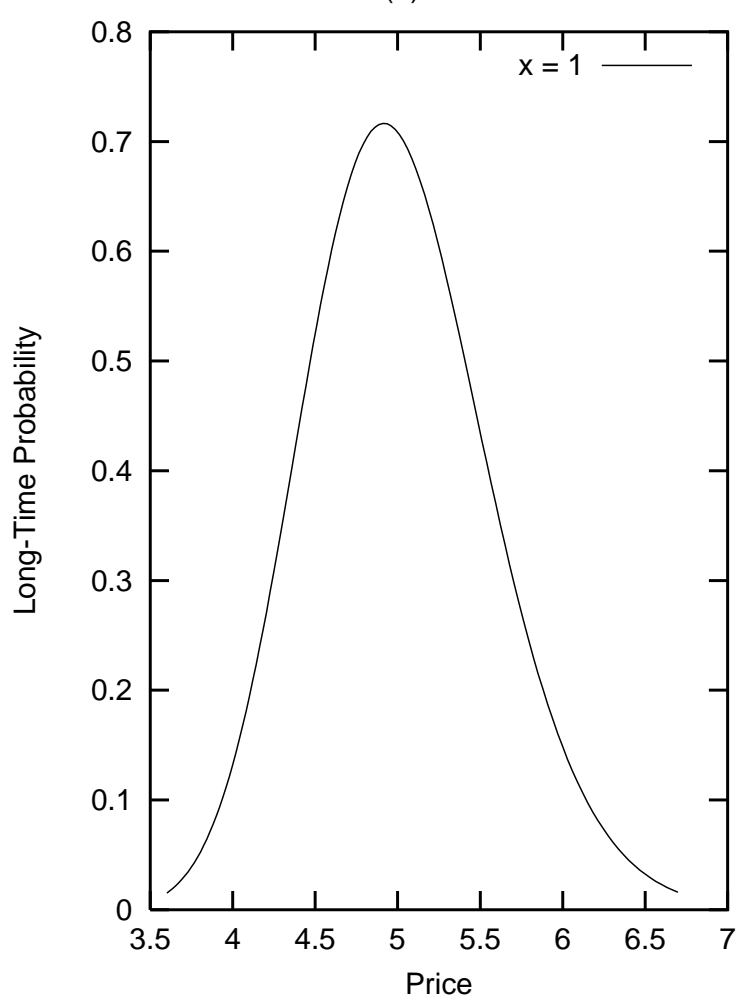

(c) $x=-1$

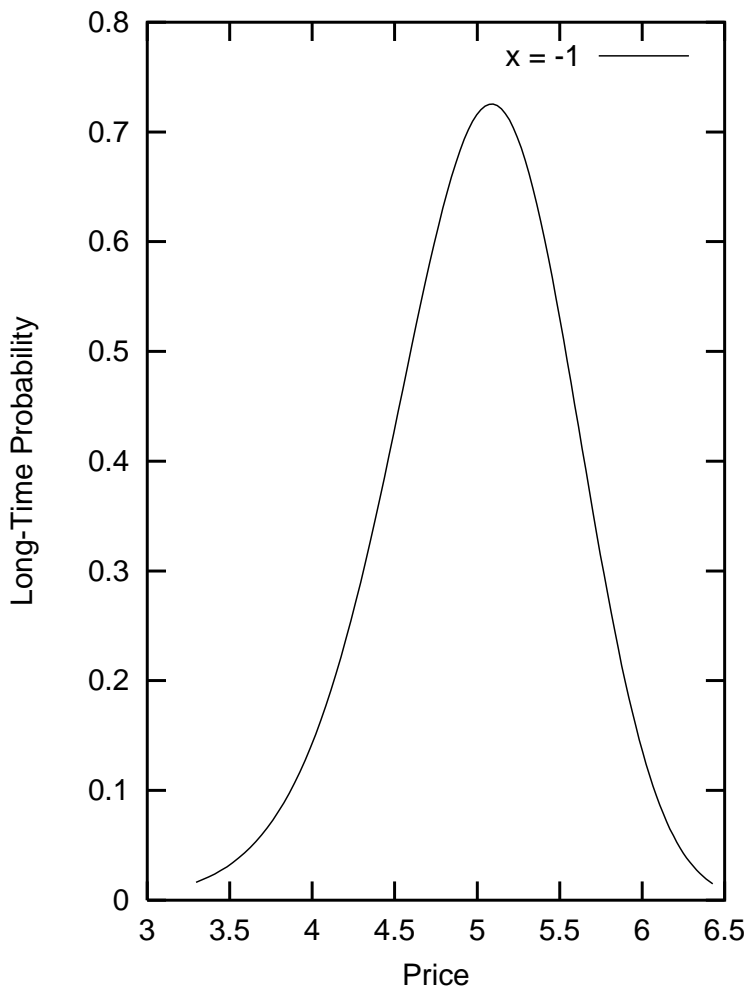

(b) $x=0$

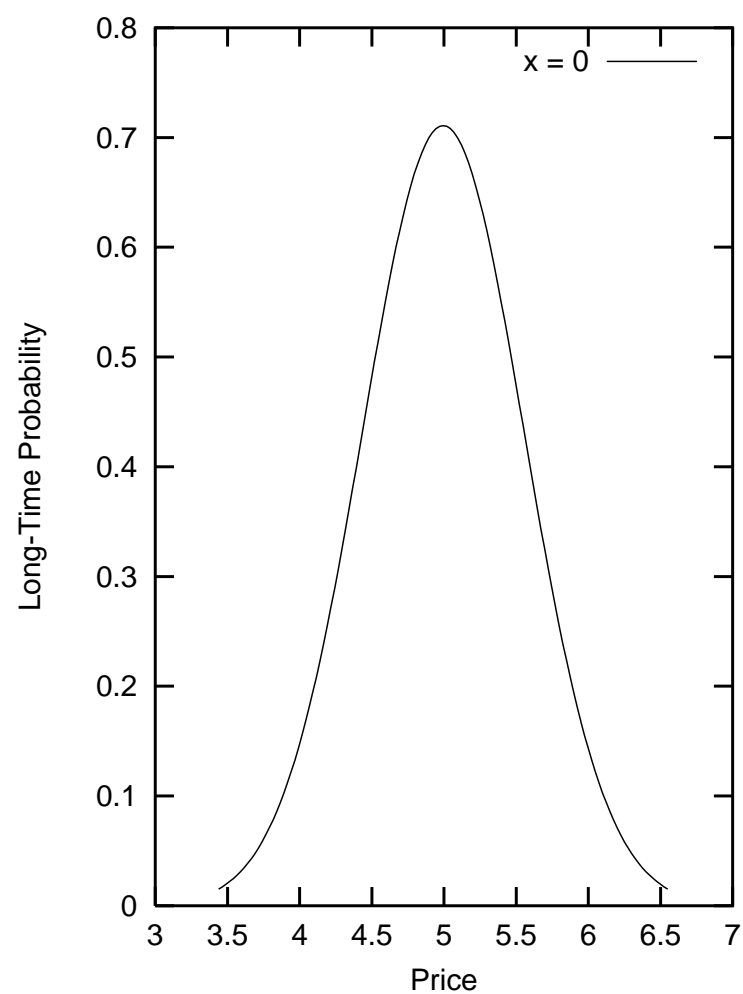

(d) $x=2$

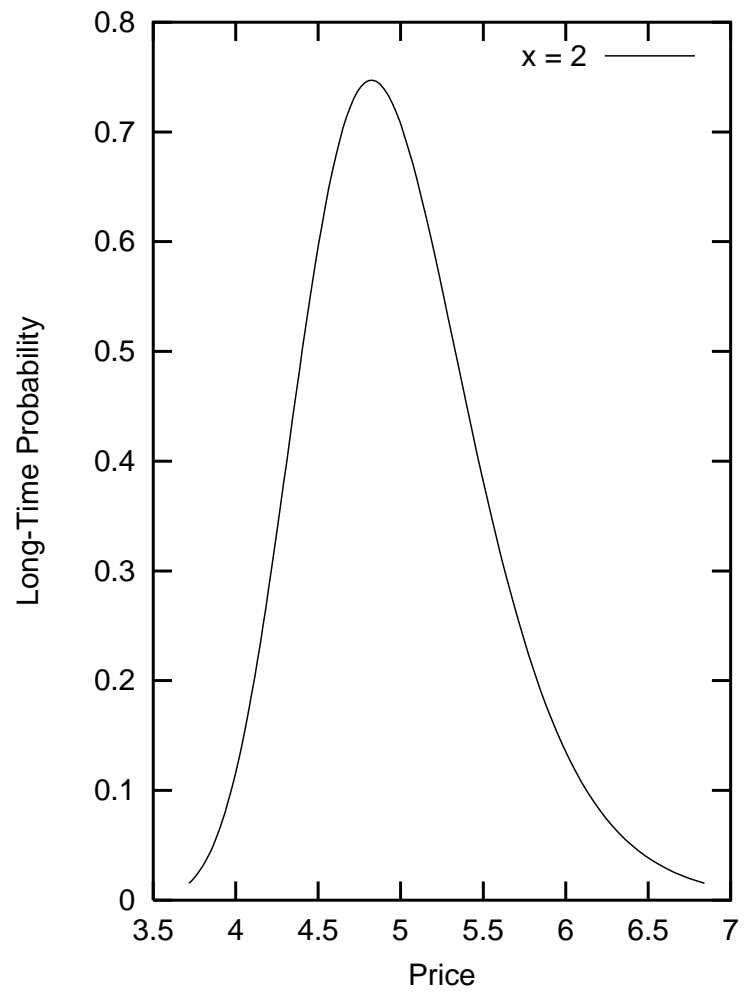


Long-Time Probability

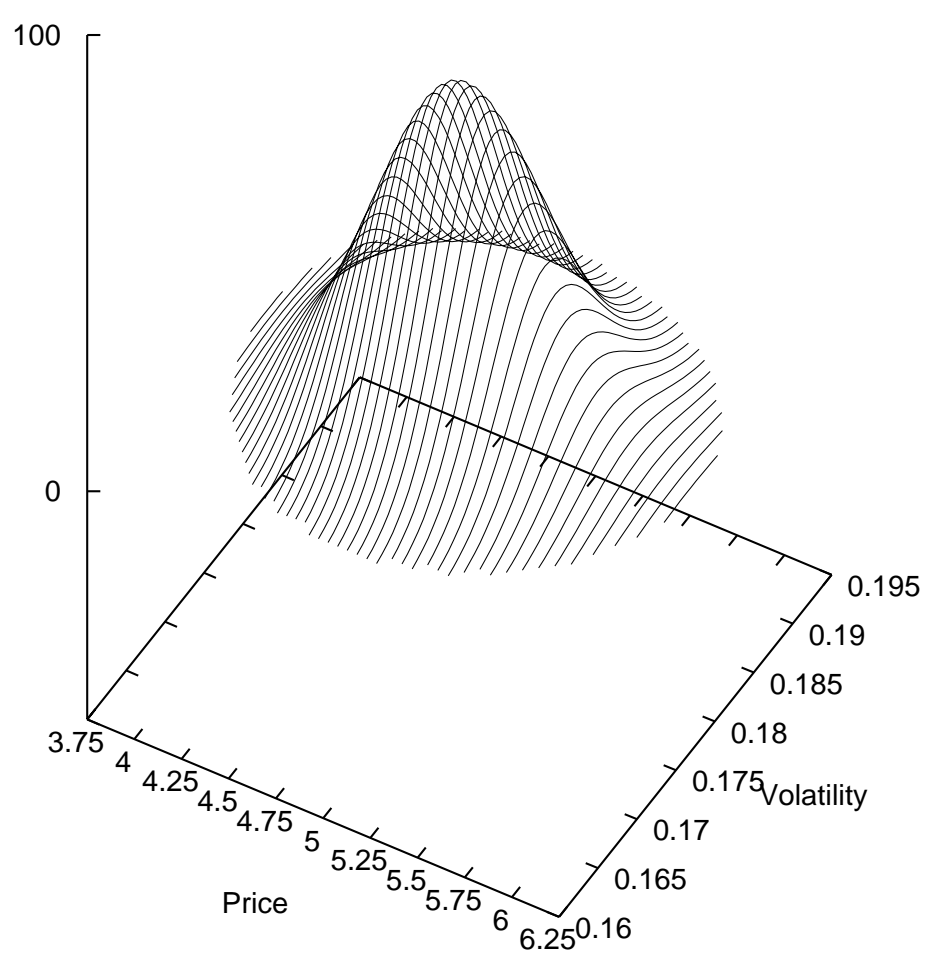




\begin{tabular}{l|ccccc} 
Greek & $\mathrm{BS}$ & $\mathrm{CRR}_{\text {European }}$ & $\mathrm{CRR}_{\text {American }}$ & $\mathrm{CRR}_{\text {variant }}$ & PATHINT \\
\hline \hline Price & 0.138 & 0.138 & 0.138 & 0.138 & 0.138 \\
Delta & 0.501 & 0.530 & 0.534 & 0.506 & 0.501 \\
Gamma & 1.100 & 1.142 & 1.159 & 1.116 & 1.100 \\
Theta & -0.131 & -0.130 & -0.132 & -0.133 & -0.131 \\
Rho & -0.0688 & -0.0688 & -0.0530 & -0.0530 & -0.0688 \\
Vega & 1.375 & 1.375 & 1.382 & 1.382 & 1.375
\end{tabular}

